\title{
ANALYSIS AND SIMULATIONS OF THE CHEN-LUBENSKY ENERGY FOR SMECTIC LIQUID CRYSTALS: ONSET OF UNDULATIONS*
}

\author{
CARLOS J. GARCíA-CERVERA ${ }^{\dagger}$ AND SOOKYUNG JOO
}

\begin{abstract}
We study the Chen-Lubensky energy to investigate layer undulations in smectic liquid crystals in response to an applied magnetic field. In earlier work [C.J. García-Cervera and S. Joo, Arch. Rat. Mech. Anal., 203(1), 1-43, 2012], the authors obtained an asymptotic expression of the unstable modes and a sharp estimate of the critical field using the Landau-de Gennes model for smectic A liquid crystals. In this paper, we extend our theory to the Chen-Lubensky energy, which includes a second order smectic order parameter gradient. Analysis based on $\Gamma$-convergence theory and bifurcation theory provide the estimate of the critical field and frequency of the undulations. Furthermore, we present a new numerical formulation of fourth order partial differential equations. With this formulation, the fourth order system reduces to a second order equation with a constraint, which resembles the incompressible Navier-Stokes equations from fluid dynamics. We use this method to illustrate the presence of layer undulations near the critical field and to confirm that the results from our analysis agree with these numerical simulations. We also use asymptotic analysis to determine the structure of the domain wall at high fields under the assumption that the layer density is constant.
\end{abstract}

Key words. Chen-Lubensky, smectic liquid crystals, undulations.

AMS subject classifications. 65M06, 35B30, 35B35, 34E10.

\section{Introduction}

We study the formation of undulations under an applied magnetic field in smectic liquid crystals using the Chen-Lubensky energy functional. We consider a smectic liquid crystal confined between two flat plates and uniformly aligned in a way that the smectic layers are parallel to the bounding plates. The external magnetic field is applied in the direction parallel to the smectic layers. If the field reaches the critical threshold, the layers undulate. This phenomenon is called the Helfrich-Hurault effect (see [18] and [20]). In our previous work [15], we studied this phenomenon analytically by considering the minimizer of the second variation of the Landau-de Gennes free energy $([7])$ at the undeformed state and by carrying out a bifurcation analysis of the nontrivial solution curve. In this paper we study undulation phenomena with the modified Chen-Lubensky energy that includes the second order gradient term. This energy can describe both smectic A and smectic C phases depending on the temperature-dependent material parameter.

The classic Helfrich-Hurault theories assume that the layers are fixed at the cell boundaries, i.e., that the undulations vanish at the boundaries and a specific form of an ansatz is made. However, experimental studies of undulations ([21, 28]) showed a lower critical field and larger layer displacement than the ones expected from the HelfrichHurault theory. Furthermore, the displacement of the layer undulations immediately adjacent to the bounding plates was not zero. In [15], we analyzed the de Gennes free

${ }^{*}$ Received: October 21, 2012; accepted (in revised form): August 22, 2013. Communicated by Pingwen Zhang.

${ }^{\dagger}$ Mathematics Department, University of California, Santa Barbara, CA 93106, USA (cgarcia@math.ucsb.edu).

This author's research was supported by NSF grants DMS-0645766 and DMS-0908538.

${ }^{\ddagger}$ Department of Mathematics and Statistics, Old Dominion University, Norfolk, VA 23529, USA (sjoo@odu.edu).

This author's research was supported by NSF grant DMS-0908538. 
energy with and without fixing the layers on the boundaries. In this paper, we extend the analysis used in [15] to the Chen-Lubensky functional for smectic A liquid crystals and find that the same critical fields and undulation descriptions are obtained with both boundary conditions. Thus without the assumption that the layers are fixed at the boundaries, a lower critical field and a description of the undulations consistent with experiments are successfully achieved.

We apply $\Gamma$-convergence theory to a one dimensional energy that characterizes the critical field, and consider both Dirichlet and Natural boundary conditions on the layer variable. For this, we identify a small parameter while performing nondimensionalization of the energy. The same $\Gamma$-limit in [15] is given with the Chen-Lubensky energy. We also study the Euler-Lagrange equations of the Chen-Lubensky energy via bifurcation theory. We prove that there is a pitchfork bifurcating curve of nontrivial solutions above the critical magnetic field. The stability of the undeformed state is lost at the bifurcation point. Furthermore, a sharp estimate of the critical field and a description of the undulation phenomena are given analytically for smectic A liquid crystals.

In sections 2 and 3, we present the modified Chen-Lubensky model and introduce an efficient numerical method for the gradient flow of the Chen-Lubensky functional and study the Helfrich-Hurault effect in smectic liquid crystals. The numerical method is based on a new formulation of the resulting fourth order partial differential equations. With this formulation, the system reduces to second order equations with a constraint, which resembles the Navier-Stokes equations from fluid dynamics.

To illustrate the new approach, consider a functional of the form

$$
F(\psi)=\int_{\Omega} f\left(\nabla \psi, \nabla^{2} \psi\right) d \mathbf{x}
$$

Its gradient flow results in a fourth order partial differential equation. Instead, we define $\mathbf{m}=\nabla \psi$, and rewrite the energy in terms of $\mathbf{m}$ :

$$
F(\mathbf{m})=\int_{\Omega} f(\mathbf{m}, \nabla \mathbf{m}) d \mathbf{x} .
$$

The corresponding gradient flow equation becomes

$$
\frac{\partial \mathbf{m}}{\partial t}=\nabla \cdot \frac{\partial f}{\partial \nabla \mathbf{m}}-\frac{\partial f}{\partial \mathbf{m}}-\nabla^{\perp} p
$$

where the last term $\nabla^{\perp} p=\left(-\partial_{y} p, \partial_{x} p\right)$ appears as a Lagrange multiplier for the constraint $\nabla \times \mathbf{m}=0$, in a similar way as the pressure plays the role of a Lagrange multiplier for the incompressibility constraint in the Navier-Stokes equations. In fact, if we define $\mathbf{u}=\mathbf{m}^{\perp}=\left(-m_{2}, m_{1}\right)$, then the same argument leads to the equation

$$
\frac{\partial \mathbf{u}}{\partial t}=\nabla \cdot \frac{\partial f}{\partial \nabla \mathbf{u}}-\frac{\partial f}{\partial \mathbf{u}}-\nabla p
$$

which shows more clearly the resemblance with the Navier-Stokes equations of fluid dynamics. A large number of numerical methodologies have been developed for this equation, and we adapt here the Gauge method, introduced in [9] to solve the NavierStokes equations, which allows for an efficient treatment of the pressure term.

We illustrate the layer undulation patterns numerically by solving the gradient flow of smectic A liquid crystals. We find good agreement with the estimate of the 
critical field and the frequencies of layer undulation obtained in sections 5 and 6 . Numerical simulations and analysis for undulations in smectic A liquid crystals are presented in [15] when $D=0$ in the Chen-Lubenksy energy. The influence of an applied magnetic field in smectic $\mathrm{C}$ liquid crystals in a two dimensional domain is illustrated as well. When the directors at the cell boundaries are tilted with a fixed angle $\alpha$, the director tends to align with the magnetic field as expected. However, the simulation shows that the layers are not disturbed regardless of the strength of the magnetic field. When the director is arranged with a tilt angle $\alpha$ at the top and $-\alpha$ at the bottom of the cell, the layers are uniformly aligned at a moderate field and start to undulate at a higher field.

In Section 4, we study the stability of the undeformed state of smectic A liquid crystals. We use a simplified model of constant layer density. The case of variable layer density was considered for the de Gennes energy in [15]. We define the critical field $H_{c}$ above which the undeformed state is unstable and below which the undeformed state is stable. In order to study the limiting problem with the help of $\Gamma$-convergence theory, we reformulate the problem by taking the small parameter $\varepsilon=h^{-1}$, where $h$ is the ratio of the layer thickness to the cell thickness.

In sections 5 and 6 , we extend our study in [15] to the Chen-Lubensky functional and obtain the same results in terms of the critical field and undulation pattern. For the case where the layers are fixed at the boundary, we prove that if $2 d$ is the cell thickness, then the critical field $H_{c}$ is estimated by

$$
H_{c} \approx\left(\frac{\pi K}{\chi_{a} d \lambda}\right)^{\frac{1}{2}} .
$$

where $K, \chi_{a}$, and $\lambda$ are material constants which will be discussed in the Section 2. This estimate is consistent with the result found in the classical Helfrich-Hurault theory (see [8, p. 363] and [20]) and numerical simulation in figure 3.1 of Section 3. Furthermore, we prove that the maximum undulation occurs in the middle of the cell and the displacement amplitude decreases near the boundary. These are consistent with the result found in classic Helfrich-Hurault theory in an infinite sample. The theory also provides the period of the oscillation in the $x$ axis. The frequency of the oscillation is proportional to $d^{-1 / 2}$.

For the general case where the Dirichlet boundary condition for the layer function is eliminated, the critical threshold is lower than $H_{c}$ in (1.1) by a factor of $1 / \sqrt{\pi}$. Instead of the periodic profile as in the Dirichlet case, the director has boundary layers at both endpoints $(y= \pm d)$. The frequency of the oscillation is no longer proportional to $d^{-1 / 2}$; in fact, the frequency is of the order of $d^{-1}$.

In Section 6, we find a first eigenvalue and corresponding eigenfunctions in order to study the discrete spectrum of the linearized operator. We show that the kernel of the linearized operator at the first eigenvalue is one-dimensional for both Dirichlet and natural boundary conditions. This allows us to analyze the undulation phenomena as a bifurcation from the uniform solution. We prove that for $\kappa>\kappa_{c}$, the undeformed state loses its stability and bifurcation occurs, generating a nontrivial solution curve which is stable.

Formal asymptotic analysis is used in Section 7 to study the limiting profile of the transition wall as $\kappa \rightarrow \infty$, which gives a result consistent with the numerical simulations presented in Section 3. Well above the critical field, experiment shows that the layer density is no longer uniform, and therefore a more complex model with variable layer density needs to be used, which will be the subject of future publications. 


\section{Model}

The total free energy density for liquid crystals consists of a nematic $f_{n}$ and a smectic $f_{s}$ part. The Oseen-Frank energy density for a nematic is given by

$$
f_{n}=\frac{K_{1}}{2}(\nabla \cdot \mathbf{n})^{2}+\frac{K_{2}}{2}(\mathbf{n} \cdot \nabla \times \mathbf{n})^{2}+\frac{K_{3}}{2}|\mathbf{n} \times(\nabla \times \mathbf{n})|^{2},
$$

where $K_{1}, K_{2}$, and $K_{3}$ are the splay, twist, and bend elastic constants, respectively. We consider the energy with one constant approximation case, $K_{1}=K_{2}=K_{3}=$ $\frac{K}{2}>0$ and $K_{4}=0$. Then the nematic energy density becomes

$$
f_{n}=\frac{K}{2}|\nabla \mathbf{n}|^{2} .
$$

The smectic energy density can be expressed in terms of the nematic director $\mathbf{n}$ and the smectic order parameter

$$
\Psi(\mathbf{x})=\rho(\mathbf{x}) e^{i q \psi(\mathbf{x})},
$$

where the molecular mass density is given by

$$
\delta(\mathbf{x})=\rho_{0}(\mathbf{x})+\frac{1}{2}\left(\Psi(\mathbf{x})+\Psi^{*}(\mathbf{x})\right)=\rho_{0}(\mathbf{x})+\rho(\mathbf{x}) \cos q \psi(\mathbf{x})
$$

and $\rho_{0}$ is a locally uniform mass density, $\rho(\mathbf{x})$ is the mass density of the smectic layers, and $\psi$ parametrizes the layers so that $\nabla \psi$ is the direction of the layer normal. Now the smectic energy density is given by

$$
f_{s}=\frac{D}{2}\left|\mathbf{D}_{\mathbf{n}}^{2} \Psi\right|^{2}+\frac{C_{\perp}}{2}\left|\mathbf{D}_{\mathbf{n}} \Psi\right|^{2}+\frac{C_{\|}}{2}\left|\mathbf{n} \cdot \mathbf{D}_{\mathbf{n}} \Psi\right|^{2},
$$

where $\mathbf{D}_{\mathbf{n}}=\nabla-i q \mathbf{n}, \mathbf{D}_{\mathbf{n}}^{2}=\mathbf{D}_{\mathbf{n}} \cdot \mathbf{D}_{\mathbf{n}}$, and $D, C_{\perp}$, and $C_{\|}$are positive constants. The original model for smectic $\mathrm{C}$ energy was proposed by Chen and Lubensky in [2]. However, because their model has an anisotropic second order gradient term, we use the modified model, introduced by Luk'yanchuk [27] and analyzed in [22]. The energy $\mathfrak{F}$ is designed to describe nematic to smectic $C$ transitions if $C_{\perp}<0$ and to smectic $A$ if $C_{\perp}>0$. With this modified model, when $C_{\perp}<0$, the same tilt angle and layer thickness are obtained from the original Chen-Lubensky model [22],

$$
\tan ^{2} \alpha=\frac{-C_{\perp}}{2 D q^{2}}, \quad\left(\frac{2 \pi}{d}\right)^{2}=q^{2}+\frac{-C_{\perp}}{2 D},
$$

where $\alpha$ is the tilt angle between the director and the layer normal and $d$ is the layer thickness. Because we investigate the smectic structure far from the nematic-smectic transition, we may assume that the magnitude of the smectic order parameter is a constant, i.e., $\rho$ is a constant. Then $f_{s}$ becomes

$f_{s}=\frac{D \rho^{2} q^{4}}{2}|\nabla \psi-\mathbf{n}|^{4}+\frac{D \rho^{2} q^{2}}{2}(\Delta \psi-\nabla \cdot \mathbf{n})^{2}+\frac{C_{\perp} q^{2} \rho^{2}}{2}|\nabla \psi-\mathbf{n}|^{2}+\frac{C_{\|} q^{2} \rho^{2}}{2}(\mathbf{n} \cdot \nabla \psi-1)^{2}$.

We consider a smectic liquid crystal confined between two flat plates $(y=-d$ and $y=d)$ and a magnetic field applied in the $x$ direction.

The magnetic energy is given by $([8,29])$

$$
f_{m}=-\frac{\chi_{a}}{2}(\mathbf{n} \cdot \mathbf{H})^{2}=-\frac{\chi_{a}}{2} \sigma^{2}(\mathbf{h} \cdot \mathbf{n})^{2},
$$


where $\chi_{a}$ is the magnetic anisotropy, $\mathbf{H}=\sigma \mathbf{h}, \mathbf{h}=(1,0)$, and $\sigma=|\mathbf{H}|$. We assume that $\chi_{a}>0$. Then the director prefers to be parallel to the direction of the applied magnetic field. Collecting all contributions to the free energy, the free energy density is

$$
f=f_{n}+f_{s}+f_{m} .
$$

We consider a rectangular domain

$$
\Omega=(-L, L) \times(-d, d),
$$

where $L=r d$ for some constant $r>0$. We also assume that $\mathbf{h}=(1,0)$ so that the magnetic field tends to make the director orient along the $x$ direction. We impose periodic boundary conditions for $\phi$ and $\mathbf{n}$ in the $x$ direction, while we assume strong anchoring conditions for $\mathbf{n}$ on the boundary plates:

$$
\mathbf{n}(x, \pm d)=(0,1) \quad \text { for all } x \in[-L, L] .
$$

We make the problem dimensionless by introducing new variables

$$
(\tilde{x}, \tilde{y})=\left(\frac{x}{\lambda}, \frac{y}{\lambda}\right) \quad \text { and } \quad \varphi=\frac{\psi}{\lambda},
$$

where $\lambda=\sqrt{\frac{K}{B}}$ is of the order of the smectic layer thickness and $B=C_{\|} \rho^{2} q^{2}$ is the de Gennes compressibility constant. Then we have a non-dimensionalized energy, dropping the tilde notation,

$$
\begin{gathered}
\mathfrak{F}=\frac{B \lambda^{2}}{2} \int_{\widetilde{\Omega}}\left(|\nabla \mathbf{n}|^{2}+D_{1}(\Delta \varphi-\nabla \cdot \mathbf{n})^{2}+\frac{D_{2}}{2}|\nabla \varphi-\mathbf{n}|^{4}+c|\nabla \varphi-\mathbf{n}|^{2}\right. \\
\left.+(\mathbf{n} \cdot \nabla \varphi-1)^{2}-\kappa^{2}(\mathbf{n} \cdot \mathbf{h})^{2}\right) d \mathbf{x},
\end{gathered}
$$

where

$$
\widetilde{\Omega}=(-\widetilde{L}, \widetilde{L}) \times(-h, h), \quad h=\frac{d}{\lambda}, \quad \widetilde{L}=\frac{L}{\lambda} .
$$

The dimensionless parameters used are given by

$$
D_{1}=\frac{D \rho^{2} q^{2}}{K}, \quad D_{2}=\frac{2 D q^{2}}{C_{\|}}, \quad c=\frac{C_{\perp}}{C_{\|}}, \quad \kappa^{2}=\frac{\chi_{a} \sigma^{2}}{B} .
$$

For smectic $\mathrm{C}$ liquid crystals $\left(C_{\perp}<0\right)$, we use the following set of parameters:

$$
D_{1}=\frac{D \rho^{2} q^{2}}{K}=\frac{3.8 \times 9}{4 \pi^{2} \times 100}, \quad D_{2}=\frac{2 D q^{2}}{C_{\|}}=0.76, \quad c=\frac{C_{\perp}}{C_{\|}}=-0.1 .
$$

Then the tilt angle equation (2.1) becomes $\tan ^{2} \alpha=-c / D_{2}$. This set of model parameters was used in [32] and this choice was made so that $\alpha=20^{\circ}$ in the tilt angle equation. The first term of the second line in (2.3) often can be omitted in the description of smectic A liquid crystals where $C_{\perp}>0$. Nondimensionalization in this case leads to the dimensionless parameters

$$
\begin{aligned}
& D_{1}=\frac{D \rho^{2} q^{2}}{K} \approx 0.01, \quad D_{2}=\frac{2 D q^{2}}{C_{\perp}}=7.6, \quad c=1, \\
& \kappa^{2}=\frac{\chi_{a} \sigma^{2}}{B}, \quad B=C_{\perp} \rho^{2} q^{2}, \quad \alpha=0^{\circ} .
\end{aligned}
$$




\section{Description of numerical scheme}

One can see that the Chen-Lubensky free energy gives rise to a fourth order partial differential equation. In fact, the gradient flow equations associated with the energy (2.3) are given by

$$
\begin{aligned}
& \frac{\partial \mathbf{n}}{\partial \tau}=-\mathbf{n} \times \mathbf{n} \times\left(\Delta \mathbf{n}-D_{1} \nabla(\Delta \varphi-\nabla \cdot \mathbf{n})+D_{2}|\nabla \varphi-\mathbf{n}|^{2}(\nabla \varphi-\mathbf{n})\right. \\
& \left.+c(\nabla \varphi-\mathbf{n})-(\mathbf{n} \cdot \nabla \varphi-1) \nabla \varphi+\kappa^{2}(\mathbf{n} \cdot \mathbf{h}) \mathbf{h}\right), \\
& \frac{\partial \varphi}{\partial \tau}=-D_{1} \Delta(\Delta \varphi-\nabla \cdot \mathbf{n})+2 D_{2}\left(\partial_{j} \varphi-\mathbf{n}_{j}\right)\left(\partial_{i j} \varphi-\partial_{i} \mathbf{n}_{j}\right)\left(\partial_{i} \varphi-\mathbf{n}_{i}\right) \\
& +\left(D_{2}|\nabla \varphi-\mathbf{n}|^{2}+c\right)(\Delta \varphi-\nabla \cdot \mathbf{n})+\nabla \cdot[(\mathbf{n} \cdot \nabla \varphi-1) \mathbf{n}] .
\end{aligned}
$$

The expression $-\mathbf{n} \times \mathbf{n} \times()$ on the right hand side of the first equation results from the constraint $|\mathbf{n}|=1$. In fact, accounting for this constraint we may introduce the Lagrange multiplier $\lambda$ so that the gradient flow equation for $\mathbf{n}$ is

$$
\mathbf{n}_{t}=-\frac{\delta \mathcal{F}}{\delta \mathbf{n}}+\lambda \mathbf{n}
$$

Taking the inner product with $\mathbf{n}$ in both sides gives, and by using $|\mathbf{n}|=1$ and $\lambda=\left(\mathbf{n}, \frac{\delta \mathcal{F}}{\delta \mathbf{n}}\right),(3.2)$ becomes

$$
\mathbf{n}_{t}=\Pi_{\mathbf{n}}\left(-\frac{\delta \mathcal{F}}{\delta \mathbf{n}}\right)
$$

where $\Pi_{\mathbf{n}}(\mathbf{v})=\mathbf{v}-(\mathbf{n}, \mathbf{v}) \mathbf{n}$ is the projection of $\mathbf{v}$ onto the tangent plane to the unit sphere at $\mathbf{n}$. Equation (3.3) can be seen equivalent to the first equation of (3.1) as a consequence of the vector identity $\mathbf{u} \times(\mathbf{v} \times \mathbf{w})=(\mathbf{u}, \mathbf{w}) \mathbf{v}-(\mathbf{u}, \mathbf{v}) \mathbf{w}$. Note also that the first equation of (3.1) resembles the Landau-Lifshitz equation of micromagnetics in the high damping limit [10], and the heat-flow of harmonic maps [30].

In this section, we introduce the new formulation for the numerical simulations of Helfrich-Hurault effect for smectic liquid crystals using the Chen-Lubensky functional.Setting $\mathbf{u}=\nabla \varphi$, the energy becomes

$$
\begin{gathered}
\mathfrak{F}=\frac{B}{2} \int_{\Omega}\left(|\nabla \mathbf{n}|^{2}+D_{1}(\nabla \cdot \mathbf{u}-\nabla \cdot \mathbf{n})^{2}+\frac{D_{2}}{2}|\mathbf{u}-\mathbf{n}|^{4}+c|\mathbf{u}-\mathbf{n}|^{2}\right. \\
\left.+(\mathbf{n} \cdot \mathbf{u}-1)^{2}-\kappa^{2}(\mathbf{n} \cdot \mathbf{h})^{2}\right) d \mathbf{x}
\end{gathered}
$$

with the constraint $\nabla \times \mathbf{u}=0$.

Now the energy has only first derivatives and hence the corresponding GinzburgLandau equations are second order partial differential equations. We consider the gradient flow of the energy

$$
\begin{gathered}
\gamma_{n} \frac{\partial \mathbf{n}}{\partial t}=-\mathbf{n} \times \mathbf{n} \times\left(\Delta \mathbf{n}-D_{1} \nabla(\nabla \cdot \mathbf{u}-\nabla \cdot \mathbf{n})+\left(D_{2}|\mathbf{u}-\mathbf{n}|^{2}+c\right)(\mathbf{u}-\mathbf{n})\right. \\
\left.\quad-(\mathbf{n} \cdot \mathbf{u}-1) \mathbf{u}+\kappa^{2}(\mathbf{n} \cdot \mathbf{h}) \mathbf{h}\right), \\
\gamma_{u} \frac{\partial \mathbf{u}}{\partial t}=D_{1} \nabla(\nabla \cdot \mathbf{u}-\nabla \cdot \mathbf{n})-\left(D_{2}|\mathbf{u}-\mathbf{n}|^{2}+c\right)(\mathbf{u}-\mathbf{n})-(\mathbf{n} \cdot \mathbf{u}-1) \mathbf{n}-\nabla^{\perp} p
\end{gathered}
$$

where $\gamma_{n}$ and $\gamma_{u}$ are appropriate constants. The last term $\nabla^{\perp} p$ from the second equation arises from the constraint $\nabla \times \mathbf{u}=0$, and plays a similar role to the one 
played by the pressure in the Navier-Stokes equations. From the identity

$$
\nabla(\nabla \cdot \mathbf{u})=\Delta \mathbf{u}+\nabla \times \nabla \times \mathbf{u},
$$

and the constraint $\nabla \times \mathbf{u}=0$, we can see that $\nabla(\nabla \cdot \mathbf{u})=\Delta \mathbf{u}$. This gives a system of parabolic equations, which resembles the Navier-Stokes equations from fluid dynamics, for which a large number of numerical techniques are available $[1,3,9,16,17,26]$. The layer structure can be recovered by solving

$$
\Delta \varphi=\nabla \cdot \mathbf{u},
$$

with appropriate boundary conditions, and plays a role similar to the stream function in fluid dynamics. The contour map of $\varphi$ describes the layer undulation patterns.

This approach can have broad applicability, and can be very efficient for any functional of the form

$$
\int_{\Omega} f\left(\nabla \psi, \nabla^{2} \psi\right) d \mathbf{x}
$$

where $\Omega \subset \mathbb{R}^{2}$, and $f$ is smooth. Moreover, this formulation also can be used for any functional of the form

$$
\int_{\Omega} f\left(\psi, \nabla \psi, \nabla^{2} \psi\right) d \mathbf{x}
$$

In this case, the term $\psi$ can be obtained from $\mathbf{u}$ by solving $\Delta \psi=\nabla \cdot \mathbf{u}$ with appropriate boundary conditions, where $\mathbf{u}=\nabla \psi$. A similar situation occurs in the context of the Landau-Lifshitz equation for micromagnetics [23, 19, 13], where the stray field can be computed by solving a Poisson equation.

This approach can be extended to three dimensions also, where the Lagrange multiplier $p$ in (3.5) is replaced by a term of the form $\nabla \times \mathbf{A}$. The equation can be solved using the projection method $[3,16]$, for example, via a Hodge decomposition [31].

We use periodic boundary conditions on each end of the cell for $\mathbf{n}$ and $\mathbf{u}$, and we assume strong surface anchoring on the plate for the director $\mathbf{n}$ and Dirichlet boundary condition for $\mathbf{u}$, assuming that the layers on the boundary are fixed:

$$
\begin{aligned}
& \left.\mathbf{n}\right|_{y= \pm h}=(\sin \alpha, \cos \alpha), \\
& \left.\mathbf{u}\right|_{y= \pm h}=(0,1),
\end{aligned}
$$

where $h=\frac{d}{\lambda}, \lambda=\sqrt{\frac{K}{B}}$ is the characteristic length of the material, and $d$ is the thickness of the sample. With this boundary condition, the energy is minimized when the layers are parallel to the plates if $\kappa=0$.

We use the Gauge method [9] to solve the system (3.5) with boundary condition (3.6). Introducing two auxiliary variables, a vector field $\mathbf{a}$ and a gauge variable $\phi$ which satisfy $\mathbf{a}=\mathbf{u}-\nabla^{\perp} \phi,(3.5)$ becomes

$$
\begin{aligned}
& \frac{\partial \mathbf{n}}{\partial t}=-\mathbf{n} \times \mathbf{n} \times\left(\Delta \mathbf{n}+D_{1} \nabla(\nabla \cdot \mathbf{n})-D_{1} \Delta \mathbf{u}\right. \\
& \left.+\left(D_{2}|\mathbf{u}-\mathbf{n}|^{2}+c\right)(\mathbf{u}-\mathbf{n})+\kappa^{2} \mathbf{n} \cdot \mathbf{e}_{1}\right), \\
& \frac{\partial \mathbf{a}}{\partial t}=D_{1} \Delta \mathbf{a}-D_{1} \nabla(\nabla \cdot \mathbf{n})+\left(D_{2}|\mathbf{u}-\mathbf{n}|^{2}+c\right)(\mathbf{n}-\mathbf{u}),
\end{aligned}
$$




$$
\begin{aligned}
\Delta \phi & =\nabla \times \mathbf{a}, \\
\mathbf{u} & =\mathbf{a}-\nabla^{\perp} \phi,
\end{aligned}
$$

with boundary conditions

$$
\begin{aligned}
& \left.\mathbf{n}\right|_{y= \pm h}=(\sin \alpha, \cos \alpha), \\
& \left.\mathbf{a}\right|_{y= \pm h}=(\sin \alpha, \cos \alpha)+\left(0, \phi_{x}\right), \\
& \left.\frac{\partial \phi}{\partial y}\right|_{y= \pm h}=0 .
\end{aligned}
$$

A general form for this boundary condition is

$$
\frac{\partial \phi}{\partial \nu}=0, \quad \mathbf{a} \cdot \tau=\mathbf{u} \cdot \tau, \quad \mathbf{a} \cdot \nu=\mathbf{u} \cdot \nu+\frac{\partial \phi}{\partial \tau} .
$$

3.1. Smectic A liquid crystals. For smectic A liquid crystals, we set $\alpha=0$ in (3.11) and $c=1$. As described in Section 2, we impose strong anchoring conditions for the director field, and Dirichlet boundary condition on $\varphi$ at the top and the bottom plates, (3.11). Periodic boundary conditions are imposed for both $\mathbf{n}$ and $\varphi$ on each side of the domain. For the time discretization of (3.7) we use a projection method, similar to the one introduced for micromagnetics in [10]. We write $\mathbf{n}=\left(n_{1}, n_{2}\right)$ and $\mathbf{u}=\left(u_{1}, u_{2}\right)$. To update the solution, we use a fractional step approach. First, assuming that $\left(\mathbf{n}^{k-1}, \mathbf{u}^{k-1}, \phi^{k-1}\right)$ and $\left(\mathbf{n}^{k}, \mathbf{u}^{k}, \phi^{k}\right)$ are known, we solve for $\mathbf{n}^{*}$ :

$$
\begin{aligned}
\frac{\mathbf{n}^{*}-\mathbf{n}^{k}}{\Delta t}=\Delta \mathbf{n}^{*} & +D_{1}\left(\begin{array}{c}
\left(n_{1}^{*}\right)_{x x}+\left(n_{2}^{k}\right)_{x y} \\
\left(n_{1}^{*}\right)_{x y}+\left(n_{2}^{*}\right)_{y y}
\end{array}\right)-D_{1} \Delta \mathbf{u}^{k} \\
& +\left(D_{2}\left|\mathbf{u}^{k}-\mathbf{n}^{k}\right|^{2}+1\right)\left(\mathbf{u}^{k}-\mathbf{n}^{k}\right)+\kappa^{2} \mathbf{n}^{*} \cdot \mathbf{e}_{1},
\end{aligned}
$$

where $\Delta t$ is the time step size, with the boundary condition

$$
\left.\mathbf{n}^{*}\right|_{y= \pm h}=(0,1)
$$

Then we project $\mathbf{n}^{*}$ onto $\mathbb{S}^{2}$ to obtain $\mathbf{n}^{k+1}$,

$$
\mathbf{n}^{k+1}=\frac{1}{\left|\mathbf{n}^{*}\right|} \mathbf{n}^{*}
$$

For equation (3.8), we write

$$
\begin{aligned}
\frac{\mathbf{a}^{k+1}-\mathbf{a}^{k}}{\Delta t}= & D_{1} \Delta \mathbf{a}^{k+1}-D_{1}\left(\begin{array}{c}
\left(n_{1}^{k+1}\right)_{x x}+\left(n_{2}^{k+1}\right)_{x y} \\
\left(n_{1}^{k+1}\right)_{x y}+\left(n_{2}^{k+1}\right)_{y y}
\end{array}\right) \\
& +\left(D_{2}\left|\mathbf{u}^{k}-\mathbf{n}^{k+1}\right|^{2}+1\right)\left(\mathbf{n}^{k+1}-\mathbf{u}^{k}\right)
\end{aligned}
$$

with boundary condition

$$
\left.\mathbf{a}^{k+1}\right|_{y= \pm h}=\left.\left(0,1+\phi_{x}^{k}\right)\right|_{y= \pm h} .
$$

Then we obtain the gauge field (3.9) by solving

$$
\Delta \phi^{k+1}=\nabla \times \mathbf{a}^{k+1}
$$


with homogeneous Neumann boundary condition $\left.\phi_{y}^{k+1}\right|_{y= \pm h}=0$ and then update $\mathbf{u}$,

$$
\mathbf{u}^{k+1}=\mathbf{a}^{k+1}-\nabla^{\perp} \phi^{k+1}=\mathbf{a}^{k+1}+\left(\begin{array}{c}
\phi_{y}^{k+1} \\
-\phi_{x}^{k+1}
\end{array}\right) .
$$

Periodic boundary conditions are imposed for both $\mathbf{n}$ and $\varphi$ on each side of the domain. We use a Fourier spectral discretization in the $x$ direction, and second order finite differences in the $y$ direction. The fast Fourier transform is computed using the FFTW libraries [11].

The method (3.12)-(3.16) is second order accurate in space, and first order accurate in time due to the first order accuracy of the projection method (3.12)-(3.13). One can improve this to second order accuracy by employing the method also introduced in [10], however, this is not pursued in this paper. The consistency and convergence of the projection and gauge methods are given in [10] and [33], respectively. Our system contains additional nonlinear terms, but they are only lower order terms, and they are treated explicitly. Hence the consistency of our scheme can be shown in exactly the same way as in [10] and [33].

We try to find the maximum $\Delta t$ corresponding to the various $\Delta x$ by reducing $\Delta x$ from $\Delta x=0.625$ to $\Delta=0.02$. In all cases, the table 3.1 shows that the maximum $\Delta t$ is 0.4 , which gives unconditional stability for the scheme (3.12)-(3.16). For the table below, we use a domain size $20 \times 5$ and $\Delta y=0.5 \cdot \Delta x$.

\begin{tabular}{ccccccc}
\hline$\Delta x$ & 0.02 & 0.039 & 0.078 & 0.156 & 0.3125 & 0.625 \\
\hline $\operatorname{Max} \Delta t$ & 0.4 & 0.4 & 0.4 & 0.4 & 0.4 & 0.4 \\
\hline
\end{tabular}

TABLE 3.1. Stability constraint of $\Delta t$ with various $\Delta x$.

For the initial condition, we consider a small perturbation from the undeformed state. More precisely, for all $(x, y) \in \Omega$,

$$
\begin{aligned}
& \mathbf{n}(x, y, 0)=\frac{\left(\epsilon n_{1}, 1+\epsilon n_{2}\right)}{\left|\left(\epsilon n_{1}, 1+\epsilon n_{2}\right)\right|}, \\
& \varphi(x, y, 0)=y+\epsilon \varphi_{0},
\end{aligned}
$$

where a small number $\epsilon=0.01$ and $n_{1}, n_{2}$, and $\varphi_{0}$ are arbitrarily chosen.

We take the domain size $L=50$ and $h=12.5$. A more physically related value for $h$ in smectic A liquid crystals is $5 \times 10^{5}$. However, the layer undulations can be observed if $h \gg 1$. In fact, the undulations in cholesteric liquid crystals occur with $h \approx 5$ ([28]). The numbers of grid points in the $x$ and $y$ directions are both 1024 and 512 , respectively.

In figure 3.1 we show the layer structures obtained in response to the various magnetic field strengths $\kappa$. The figures are contour maps of $\varphi$ because the level sets of $\varphi$ represent the layer. One can see that the undeformed state is stable before the magnetic field $\kappa$ reaches the threshold $\kappa_{c}$ (figure 3.1 (a)). If $\kappa$ increases and reaches $\kappa_{c}$, the layer undulations occur (figure 3.1 (b)). As $\kappa$ increases beyond $\kappa_{c}$, the displacement amplitude increases as in the figure 3.1. The maximum undulation occurs in the middle of the cell $(y=0)$ and the displacement amplitude decreases when approaching the boundary $(y= \pm h)$. 

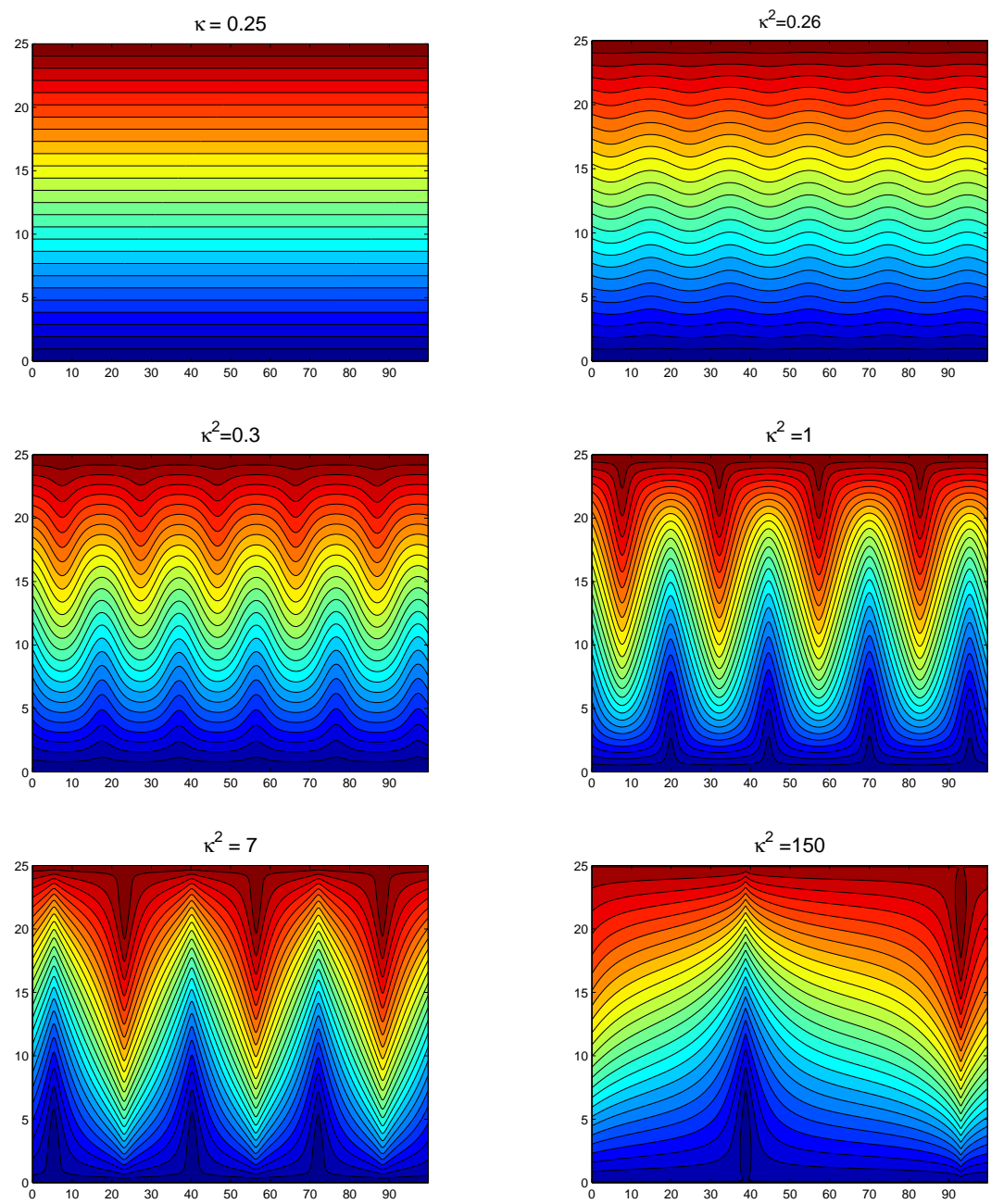

FIG. 3.1. Smectic A undulations: Contour plots of $\varphi$, the solution of the system (3.5).

Figure $3.1(\mathrm{~b})$ shows that $\kappa_{c}^{2}$ is between 0.25 and 0.26 . In fact, $\kappa_{c}^{2} \sim \pi / h$ will be proved in the sections 5 and 6 . This estimate from our analysis predicts the undulations at $\kappa_{c}^{2} \sim \pi / 12.5 \sim 0.2513$, which is a good agreement with our simulations.

In [15], the simulation using de Gennes energy shows that the undulation period decreases as the applied field increases. However, the period increases when we use the Chen-Lubensky functional as in figure 3.1. This is expected because the ChenLubensky functional includes the second order derivative term, which penalizes the changes of the gradients.

Physically relevant undulation patterns at a higher field can also be observed with this model without the assumption that the layer density is constant. This will appear in a future publication.

3.2. Smectic C liquid crystals. We consider a two dimensional domain for undulations in smectic $\mathrm{C}$ liquid crystals by taking $c=-0.1$. The undeformed state is 

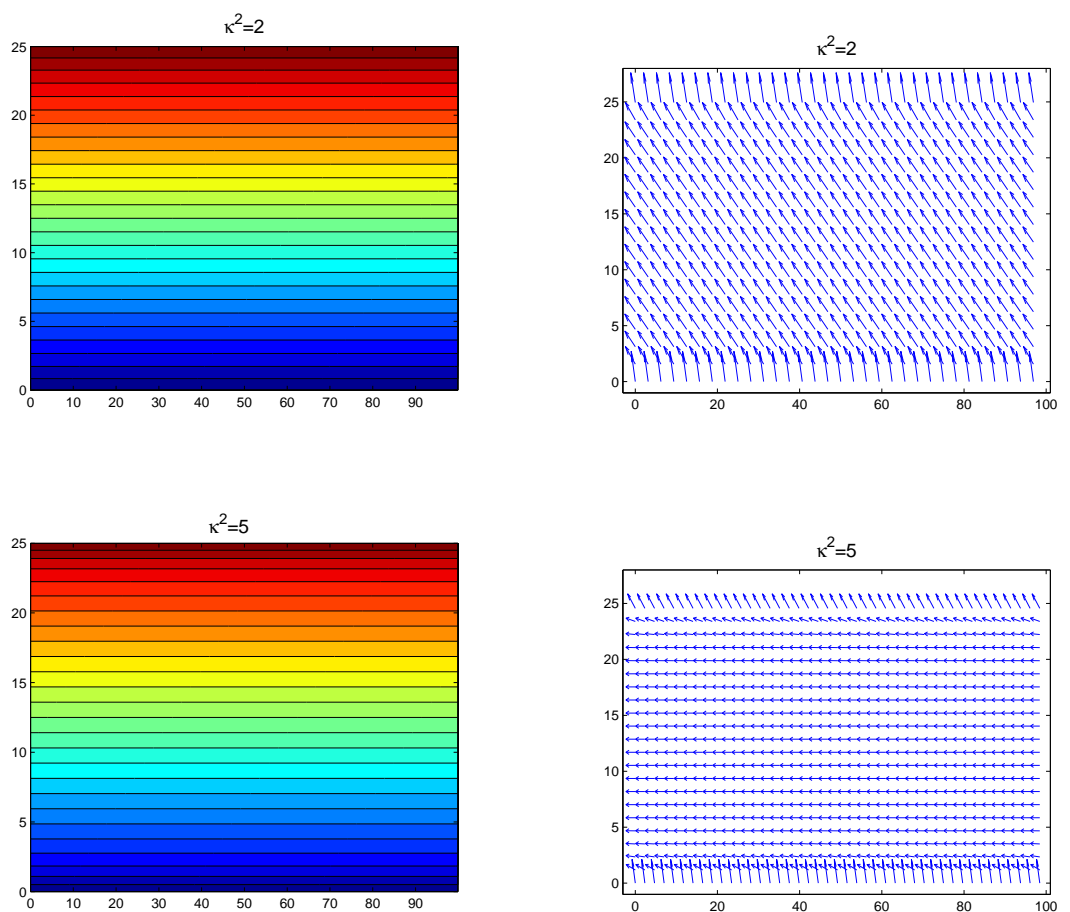

FIG. 3.2. No smectic $C$ undulations: Contour plots of $\varphi$, the solution of the system (3.5) with the same boundary condition at the top and the bottom of the cell. The first and the second column depict the layer and director, respectively.

given by

$$
\varphi_{0}=m y, \quad \text { and } \quad \theta_{0}=\alpha,
$$

where $m=\sqrt{1+\frac{c}{D_{2}}}$ is the layer thickness and $\alpha$ is the tilt angle given by the equation $\tan ^{2} \alpha=-c / D_{2}$. We find that the layer undulation does not occur when we perform the numerical simulations with the same boundary condition at the top and the bottom of the cell (3.2). Up to $\kappa^{2}=5$, one can see that the director is horizontal in most of the cell except at the boundary, but the layer is in the unperturbed state. Instead, we proceed with the skew boundary condition,

$$
\mathbf{n}(x,-h)=(\sin \alpha, \cos \alpha) \quad \text { and } \quad \mathbf{n}(x, h)=(-\sin \alpha, \cos \alpha) .
$$

This means that the $\mathbf{c}$ director is in the positive $x$ direction at the top and is in the negative $x$ direction at the bottom of the sample cells. The numerical result obtained when $\kappa^{2}=0$ is applied was used as an initial state for numerical simulations with other values of $\kappa^{2}$. The initial state is illustrated in the first row of figure 3.3. The director changes its direction gradually to satisfy the boundary values of the top and the bottom plates. Between $\kappa^{2}=0.1$ and $\kappa^{2}=0.3$, the simulations for layers illustrate the undeformed state. As $\kappa$ increases further, one can see periodic layer oscillations as in the last row of figure 3.3 . 

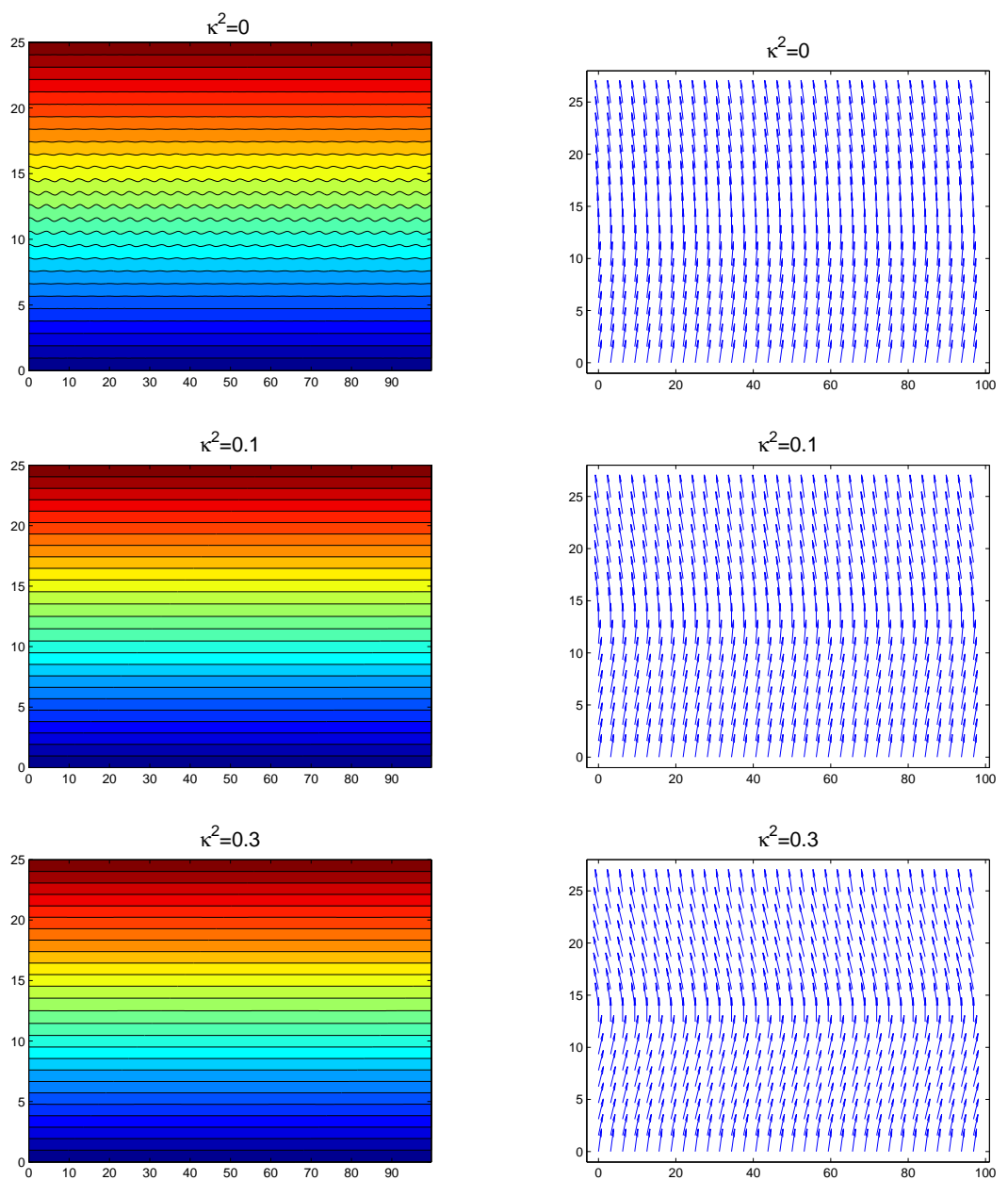

$\kappa^{2}=0.5$

$\kappa^{2}=0.5$
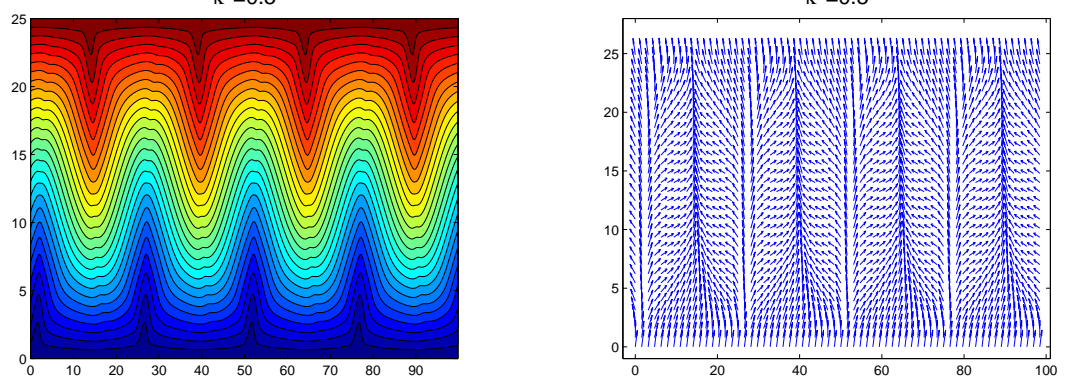

FIG. 3.3. Smectic C undulations: Contour plots of $\varphi$, the solution of the system (3.5) with the boundary condition (3.17). The first and the second column depict the layer and director, respectively. 


\section{Critical field for smectic a liquid crystals}

We derive here a one-dimensional energy that characterizes the critical field, in a similar way to the procedure described in Section 3 of [15]. For sections 4-6, we only consider smectic A liquid crystals. From $|\mathbf{n}|=1$, we can introduce the scalar variable $\theta$, with $0 \leq \theta<2 \pi$, such that

$$
\mathbf{n}=(\sin \theta, \cos \theta)
$$

Then the free energy (2.3) becomes, omitting the first term of the second line in (2.3), using (2.4), and writing simply $\kappa$ for $\kappa^{2}$,

$$
\begin{aligned}
\mathfrak{F}(\theta, \varphi)=\int_{\Omega}\left(D_{1}(\Delta \varphi\right. & \left.-\cos \theta \theta_{x}+\sin \theta \theta_{y}\right)^{2} \\
& +\frac{D_{2}}{2}\left(\left(\varphi_{x}-\sin \theta\right)^{2}+\left(\varphi_{y}-\cos \theta\right)^{2}\right)^{2}+\left(\varphi_{x}-\sin \theta\right)^{2} \\
& \left.+\left(\varphi_{y}-\cos \theta\right)^{2}+|\nabla \theta|^{2}-\kappa \sin ^{2} \theta\right) d y d x
\end{aligned}
$$

and the corresponding boundary condition on $\theta$ is the homogeneous Dirichlet boundary condition on the top and the bottom of the plate. This energy (4.1) has a trivial critical point, $\theta=0, \phi=y$, which describes the undeformed state where the layers are parallel to the boundary plates, the directors are aligned in the $y$ direction, and the magnetic filed is applied in the direction perpendicular to the director. The second variation of the energy at the undeformed state, $\phi_{0}=y$, and $\theta_{0}=0$, gives

$$
\begin{aligned}
& \frac{1}{2} D^{2} \mathfrak{F}\left(\theta_{0}+t \theta, \varphi_{0}+t \varphi\right):=\left.\frac{1}{2} \frac{d^{2}}{d t^{2}}\right|_{t=0} \mathfrak{F}\left(\theta_{0}+t \theta, \varphi_{0}+t \varphi\right) \\
= & \int_{\Omega}\left(D_{1}\left(\Delta \varphi-\theta_{x}\right)^{2}+\left(\varphi_{x}-\theta\right)^{2}+\varphi_{y}^{2}+|\nabla \theta|^{2}-\kappa|\theta|^{2}\right) d x d y .
\end{aligned}
$$

The undeformed state, $\left(\theta_{0}, \phi_{0}\right)$, is stable if the second variation is nonnegative at $\left(\theta_{0}, \phi_{0}\right)$. We set

$$
\mathcal{G}(\theta, \varphi):=\int_{\Omega}\left(D_{1}\left(\Delta \varphi-\theta_{x}\right)^{2}+\left(\varphi_{x}-\theta\right)^{2}+\varphi_{y}^{2}+|\nabla \theta|^{2}\right) d x d y
$$

and consider two admissible sets, $\mathcal{A}$, and $\mathcal{A}_{0}$, defined by

$$
\mathcal{A}=\left\{(\theta, \varphi) \in H^{1}(U) \times H^{2}(U):\|\theta\|_{2}=1, \theta(x, \pm h)=0 \text { for all } x\right\} .
$$

and

$$
\mathcal{A}_{0}=\{(\theta, \varphi) \in \mathcal{A}: \varphi(x, \pm h)=0 \text { for all } x\},
$$

respectively, where $U=\mathbb{R} /(-\widetilde{L}+2 \widetilde{L} \mathbb{Z}) \times(-h, h)$. Note that because $\varphi$ is the layer perturbation, when $D_{1}=D_{2}=0$, the set $\mathcal{A}_{0}$ corresponds to the setting in the classic Helfrich-Hurault theory, where the layers are fixed at the cell boundaries.

One can see from (4.2) that the critical field in the Neumann and Dirichlet case, $\kappa_{c}$ and $\kappa_{c}^{0}$, is defined by, respectively,

$$
\kappa_{c}=\inf _{(\theta, \varphi) \in \mathcal{A}} \mathcal{G}(\theta, \varphi) \quad \text { and } \quad \kappa_{c}^{0}=\inf _{(\theta, \varphi) \in \mathcal{A}_{0}} \mathcal{G}(\theta, \varphi) \text {. }
$$


Thus, the undeformed state, $\left(\theta_{0}, \varphi_{0}\right)$, is stable if $\kappa<\kappa_{c}^{(0)}$ and unstable if $\kappa>\kappa_{c}^{(0)}$. In addition, we show that when $\kappa=\kappa_{c}^{(0)}$, a stable bifurcation is possible.

The periodic boundary conditions allow us to use the Fourier series representation,

$$
\theta(x, y)=\sum_{n=-\infty}^{\infty} \theta_{n}(y) e^{i \mu_{n} x} \quad \text { and } \quad \varphi(x, y)=\sum_{n=-\infty}^{\infty} \varphi_{n}(y) e^{i \mu_{n} x}
$$

where $\mu_{n}=\pi n / \widetilde{L}$. Then (4.3) becomes

$$
\begin{aligned}
\mathcal{G}(\theta, \varphi)=2 \widetilde{L} \int_{-h}^{h}\left(\sum _ { n = - \infty } ^ { \infty } \left(\left|\theta_{n}^{\prime}\right|^{2}\right.\right. & +\mu_{n}^{2}\left|\theta_{n}\right|^{2}+D_{1}\left|\varphi_{n}^{\prime \prime}-\mu_{n}^{2} \varphi-i \mu_{n} \theta_{n}\right|^{2} \\
& \left.\left.+\left|\theta_{n}-i \mu_{n} \varphi_{n}\right|^{2}+\left|\varphi_{n}^{\prime}\right|^{2}\right)\right) d y
\end{aligned}
$$

and the critical field can be obtained by

$$
\begin{aligned}
\kappa_{c}^{(0)} & =\inf _{\mu_{n}} \inf _{(\theta, \phi) \in \mathcal{B}^{(0)}} \int_{-h}^{h}\left(\left|\theta^{\prime}\right|^{2}+\mu^{2}|\theta|^{2}+D_{1}\left|\varphi_{n}^{\prime \prime}-\mu_{n}^{2} \varphi-i \mu_{n} \theta_{n}\right|^{2}\right. \\
& \left.\quad+|\theta-i \mu \varphi|^{2}+\left|\varphi^{\prime}\right|^{2}\right) d y \\
& =\inf _{\mu} \inf _{(\theta, \phi) \in \mathcal{B}^{(0)}} \mathcal{F}(\theta, \varphi, \mu),
\end{aligned}
$$

where

$$
\begin{aligned}
\mathcal{B} & =\left\{(\theta, \varphi) \in W_{0}^{1,2}(-h, h) \times W^{2,2}(-h, h): \int_{-h}^{h}|\theta(y)|^{2} d y=1\right\}, \\
\mathcal{B}^{0} & =\{(\theta, \varphi) \in \mathcal{B}: \varphi( \pm h)=0\},
\end{aligned}
$$

and here we let $\mu=\mu_{n}$ for simplicity.

In the case of $D_{1}=D_{2}=0$, we showed in [14] that if $L \geq h \geq 1$ then there exist universal constants $c_{1}$ and $c_{2}$ such that

$$
\frac{c_{1}}{h} \leq \kappa_{c}^{(0)} \leq \frac{c_{2}}{h}
$$

For the general case with arbitrary material constants, the lower bound can follow exactly from the Lemma 3.2 from [14]. Also, the test functions for the upper bound in [14] can be applied for the general setting as well. Thus we have the same estimate (4.8) on the critical field.

Introducing the transformations, $x=\tilde{x} / h, y=\tilde{y} / h$, and $u=\varphi / h$, the energy in (4.1) becomes

$$
\begin{aligned}
\varepsilon \mathfrak{F}(\theta, u)=\int_{D}\left(D_{1} \varepsilon\right. & \left(\Delta u-\cos \theta \theta_{x}+\sin \theta \theta_{y}\right)^{2} \\
& +\frac{D_{2}}{2 \varepsilon}\left(\left(u_{x}-\sin \theta\right)^{2}+\left(u_{y}-\cos \theta\right)^{2}\right)^{2} \\
& \left.+\frac{1}{\varepsilon}\left(u_{x}-\sin \theta\right)^{2}+\frac{1}{\varepsilon}\left(u_{y}-\cos \theta\right)^{2}+\varepsilon|\nabla \theta|^{2}-\sigma \sin ^{2} \theta\right) d x d y
\end{aligned}
$$

where $D=(-r, r) \times(-1,1), r=\widetilde{L} / h$, and $\sigma=h \kappa$. As in (4.2), one can see that the critical field $\sigma_{c}$ is defined by

$$
\sigma_{c}=\inf _{(\theta, u) \in \widetilde{\mathcal{A}}} \mathcal{J}(\theta, u)
$$


where

$$
\mathcal{J}(\theta, u):=\int_{D}\left(D_{1} \varepsilon\left(\Delta u-\theta_{x}\right)^{2}+\frac{1}{\varepsilon}\left(u_{x}-\theta\right)^{2}+\frac{1}{\varepsilon} u_{y}^{2}+\varepsilon|\nabla \theta|^{2}\right) d x d y
$$

and $\widetilde{\mathcal{A}}$ is a corresponding admissible set after the transformation. It follows from (4.8) that there exist universal constants $c_{1}$ and $c_{2}$ such that

$$
c_{1} \leq \sigma_{c} \leq c_{2}
$$

Using notations

$$
\delta=\delta(\varepsilon)=\varepsilon \mu^{2}, \quad \mu=\mu_{n}=\frac{\pi n}{r}, \quad \varphi=i \mu u, \quad \text { and } \quad I=(-1,1),
$$

we define

$$
\begin{aligned}
\sigma_{c}^{(0)}= & \inf _{n} \inf _{(\theta, \varphi) \in \tilde{\mathcal{B}}^{(0)}} \int_{I}\left(\varepsilon\left|\theta^{\prime}\right|^{2}+\delta|\theta|^{2}+D_{1} \delta\left|\frac{\varepsilon}{\delta} \varphi^{\prime \prime}-\varphi+\theta\right|^{2}\right. \\
& \left.+\frac{1}{\varepsilon}|\theta-\varphi|^{2}+\frac{1}{\delta}\left|\varphi^{\prime}\right|^{2}\right) d y \\
:=\inf _{n} \inf _{(\theta, \varphi) \in \tilde{\mathcal{B}}^{(0)}} F_{\varepsilon}(\theta, \varphi, \delta), &
\end{aligned}
$$

where

$$
\begin{aligned}
\widetilde{\mathcal{B}} & =\left\{(\theta, \varphi) \in W_{0}^{1,2}(I) \times W^{2,2}(I): \int_{-1}^{1}|\theta(y)|^{2} d y=1\right\} \\
\widetilde{\mathcal{B}}^{0} & =\{(\theta, \varphi) \in \widetilde{\mathcal{B}}: \varphi( \pm 1)=0\} .
\end{aligned}
$$

From (4.12), we can see that the infimum of $F_{\varepsilon}$ is of order 1 in $\varepsilon$.

\section{5. $\Gamma$-convergence}

In this section, we look for a minimizer of $F_{\varepsilon}$ in two function spaces, $\widetilde{\mathcal{B}}$ and $\widetilde{\mathcal{B}}^{0}$, with the constraint

$$
\int_{I}|\theta(z)|^{2} d z=1
$$

via $\Gamma$-convergence theory $[6]$. We recall the definition of $\Gamma$-limit of a family of functionals.

Definition 5.1. Let $(X, \mathcal{T})$ be a topological space, and let $F_{h}$ a family of functionals parametrized by $h$. A functional $F_{0}$ is the $\Gamma$-limit of $F_{h}$ as $h \rightarrow 0$ in $\mathcal{T}$ if and only if the two following conditions are satisfied:

(i) If $u_{h} \rightarrow u_{0}$ in $\mathcal{T}$, then $\liminf _{h \rightarrow 0} F_{h}\left(u_{h}\right) \geq F_{0}\left(u_{0}\right)$.

(ii) For all $u_{0} \in X$, there exists a sequence $u_{h} \in X$ such that $u_{h} \rightarrow u_{0}$ in $\mathcal{T}$, and $\lim _{h \rightarrow 0} F_{h}\left(u_{h}\right)=F_{0}\left(u_{0}\right)$.

Condition $(i)$ is related to lower-semicontinuity, while for Condition (ii) a specific construction for the converging sequence is typically required.

Notice that if $(\theta, \varphi)$ is a minimizer of $F_{\varepsilon}$, then so is $(-\theta,-\varphi)$. Thus we consider the following spaces to identify these minimizers. We let

$$
X_{n}=\widetilde{\mathcal{B}} / \sim \quad \text { and } \quad X_{d}=\widetilde{\mathcal{B}}^{0} / \sim,
$$


where $\left(\theta_{1}, \varphi_{1}\right) \sim\left(\theta_{2}, \varphi_{2}\right)$ if and only if $\left(\theta_{2}, \varphi_{2}\right)=\left(-\theta_{1},-\varphi_{1}\right)$. We also define the following function spaces for the $\Gamma$-limit with each boundary condition,

$$
\begin{aligned}
& X_{n}^{0}=\left\{(\theta, \varphi) \in W^{1,2}(I) \times W^{2,2}(I):\|\theta\|_{L^{2}(I)}=1\right\} / \sim, \\
& X_{d}^{0}=\left\{(\theta, \varphi) \in W_{0}^{1,2}(I) \times W_{0}^{2,2}(I):\|\theta\|_{L^{2}(I)}=1\right\} / \sim .
\end{aligned}
$$

First we consider the minimizing sequences with the Dirichlet boundary condition. Let

$$
F_{\varepsilon}(\theta, \varphi, \delta):= \begin{cases}\int_{I}\left(\varepsilon\left|\theta^{\prime}\right|^{2}+\delta|\theta|^{2}+D_{1} \delta\left|\frac{\varepsilon}{\delta} \varphi^{\prime \prime}-\varphi+\theta\right|^{2}+\frac{1}{\varepsilon}|\theta-\varphi|^{2}+\frac{1}{\delta}\left|\varphi^{\prime}\right|^{2}\right) d y \\ \text { if }(\theta, \varphi, \delta) \in X_{d} \times \mathbb{R}^{+} \\ +\infty, & \text { else. }\end{cases}
$$

In [15], the $\Gamma$-convergence method was used to understand the minimizer of the linearized functional, $F_{\varepsilon}$, when $D_{1}=0$. For Dirichlet boundary condition case, the $\Gamma$-limit obtained in $[15]$ is

$$
F_{0}^{d}(\theta, \varphi, \delta):= \begin{cases}\int_{I}\left(\delta \theta^{2}+\frac{1}{\delta} \theta^{\prime 2}\right) d z, & \text { if } \theta=\varphi \in W_{0}^{1,2}(I), \\ +\infty, & \text { else. }\end{cases}
$$

One can find that this is also the $\Gamma$-limit of $F_{\varepsilon}$ for arbitrary $D_{1}$. Because the compactness property and lower bound inequality follow from the case $D_{1}=0$ in [15], we only need to establish the construction of a minimizing sequence.

Lemma 5.2. (Construction). For any $(\theta, \varphi, \delta) \in X_{d}^{0} \times \mathbb{R}^{+}$with $\theta=\varphi$ and $\int_{I}|\theta|^{2} d z=1$, there exists a sequence $\left(\theta_{j}, \varphi_{j}, \delta_{j}\right) \in X_{d} \times \mathbb{R}^{+}$with $\int_{I}\left|\theta_{j}\right|^{2} d z=1$, converging in $\left[L^{2}(I)\right]^{2} \times \mathbb{R}$ as $j \rightarrow \infty$, to $(\theta, \varphi, \delta)$, and such that

$$
\limsup _{j \rightarrow \infty} F_{\varepsilon_{j}}\left(\theta_{j}, \varphi_{j}, \delta_{j}\right)=F_{0}^{d}(\theta, \varphi, \delta) .
$$

Proof. For simplicity, we will prove $\limsup _{j \rightarrow \infty} F_{\varepsilon_{j}}\left(\theta_{j}, \varphi_{j}, \delta_{j}\right) \leq F_{0}(\theta, \varphi, \delta)$. First we suppose that $\varphi \in C_{c}^{\infty}(I)$. We take $\theta_{j}=\theta, \delta_{j}=\delta$ for all $j \geq 1$ and let $\varphi_{j}=\varphi_{h_{j}}=\varphi * \rho_{h}$ be a regularization of $\varphi$ where $\rho$ is a mollifier. We can take $h$ small such that $\varphi_{j}( \pm 1)=0$. Note that

$$
\left\|\varphi-\varphi_{h}\right\|_{2} \leq C h\left\|\varphi^{\prime}\right\|_{2} \quad \text { and } \quad\left\|\varphi_{h}^{\prime \prime}\right\| \leq \frac{C}{h}\left\|\varphi^{\prime}\right\|_{2}
$$

for some constant $C$. To see this, we find

$$
\begin{aligned}
\left|\varphi_{h}(x)-\varphi(x)\right| & =\left|\int_{|z| \leq 1} \rho(z)(\varphi(x)-\varphi(x-h z)) d z\right| \\
& \leq h \int_{|z| \leq 1} \rho(z) \int_{0}^{1}|D \varphi(x-t h z)| d t d z,
\end{aligned}
$$

and hence we find $\left\|\varphi_{h}-\varphi\right\|_{p} \leq h\|D \varphi\|_{p}$. Also,

$$
\left\|\varphi_{h}^{\prime \prime}\right\|_{2}=\left\|\varphi^{\prime}\right\|_{2}\left\|\rho_{h}^{\prime}\right\|_{1} \leq \frac{C}{h}\left\|\varphi^{\prime}\right\|_{2} .
$$


Using these estimates with $h_{j}=\varepsilon_{j}^{2 / 3}$, we have

$$
\begin{aligned}
F_{\varepsilon_{j}}\left(\theta_{j}, \varphi_{j}, \delta_{j}\right) & \leq \int_{I}\left(\frac{2 D_{1} \varepsilon_{j}^{2}}{\delta}\left|\varphi_{j}^{\prime \prime}\right|^{2}+\left(\frac{1}{\varepsilon_{j}}+2 D_{1} \delta\right)\left(\varphi-\varphi_{j}\right)^{2}+\varepsilon_{j} \theta^{\prime 2}+\delta \theta^{2}+\frac{1}{\delta} \varphi_{j}^{\prime 2}\right) \\
& \leq C\left(\varepsilon_{j}^{2 / 3}+C \varepsilon_{j}^{1 / 3}\right)\left\|\varphi^{\prime}\right\|_{2}^{2}+\delta\|\theta\|_{2}^{2}+\varepsilon_{j}\left\|\theta^{\prime}\right\|_{2}^{2}+\frac{1}{\delta} \varphi_{j}^{\prime 2}
\end{aligned}
$$

and we arrive at the conclusion. For $\varphi \in W_{0}^{1,2}(I)$, we use a density argument.

Proposition 5.3. The variational problem in $(\theta, \varphi, \delta) \in X_{d} \times \mathbb{R}^{+}$of minimizing $F_{\varepsilon}(\theta, \varphi, \delta) \Gamma$-converges in $\left[L^{2}(I)\right]^{2} \times \mathbb{R}$ as $\varepsilon \rightarrow 0$, to the variational problem in $(\theta, \varphi, \delta) \in X_{d}^{0} \times \mathbb{R}^{+}$of minimizing $F_{0}^{d}(\theta, \varphi, \delta)$, where $F_{0}^{d}$ is defined in (5.3).

The $\Gamma$-limit $F_{d}^{0}$ has a unique minimizer (up to a sign) $\left(\cos \frac{\pi}{2} y, \cos \frac{\pi}{2} y, \frac{\pi}{2}\right)$ in $X_{d}^{0} \times \mathbb{R}^{+}$and if the $\Gamma$-limit $F_{0}^{d}$ has a unique minimizer, then it follows from the $\Gamma$-convergence theory that the sequence of minimizers of $F_{\varepsilon}$ converges to the minimizer of $F_{0}^{d}[6]$. Thus, with (5.2), we have the following result.

TheORem 5.4. Let $\left(\theta_{\varepsilon}, \varphi_{\varepsilon}, \delta\right) \in X_{d} \times \mathbb{R}^{+}$be a minimizer of $F_{\varepsilon}$. For $\varepsilon=1 / h \ll 1$, we have

$$
\varepsilon \mu^{2} \approx \frac{\pi}{2}, \quad F_{\varepsilon}\left(\theta_{\varepsilon}, \varphi_{\varepsilon}, \delta\right) \approx \pi
$$

and

$$
\int_{I}\left|\varphi_{\varepsilon}(y)-\cos \frac{\pi}{2} y\right|^{2} d y \ll 1 .
$$

We look for a minimizer of $F_{\varepsilon}$ without imposing the Dirichlet boundary condition on layers. We claim that the $\Gamma$-limit is (5.7), which is the same as the one in [15]. Again the lower bound inequality can be inherited from the case $D_{1}=0$, so one only needs to regularize the minimizing sequence obtained in [15]. In order to obtain the estimate such as (5.4), we can use an extension operator, i.e., for any $J \supset I$ and for $\varphi \in W^{1,2}(I)$, one can find an extension $\tilde{\varphi} \in W_{0}^{1,2}(J)$ such that $\tilde{\varphi}=\varphi$ in $I$ and $\|\tilde{\varphi}\|_{W^{1,2}(J)} \leq C\|\varphi\|_{W^{1,2}(I)}$. Then we employ (5.4) to $\tilde{\varphi}$. Now we have the following.

Proposition 5.5. The variational problem in $(\theta, \varphi, \delta) \in X_{n} \times \mathbb{R}^{+}$of minimizing $F_{\varepsilon}(\theta, \varphi, \delta) \Gamma$-converges in $\left[L^{2}(I)\right]^{2} \times \mathbb{R}$ as $\varepsilon \rightarrow 0$, to the variational problem in $(\theta, \varphi, \delta) \in \bar{X}_{n} \times \mathbb{R}^{+} \cup\{0\}$ of minimizing $F(\theta, \varphi, \delta)$, where

$$
F(\theta, \varphi, \delta)= \begin{cases}\int_{I}\left(\delta|\theta|^{2}+\frac{1}{\delta}\left|\theta^{\prime}\right|^{2}\right) d z+\theta(1)^{2}+\theta(-1)^{2}, & \text { if } \delta \neq 0, \theta=\varphi \\ \theta(1)^{2}+\theta(-1)^{2}, & \text { if } \delta=0, \theta=\varphi=\text { constant } \\ \infty, & \text { else }\end{cases}
$$

Analogous to Theorem 5.4, we have the following result.

Theorem 5.6. Let $\left(\theta_{\varepsilon}, \varphi_{\varepsilon}, \delta_{\varepsilon}\right) \in X_{n} \times \mathbb{R}^{+}$be a minimizer of $F_{\varepsilon}$ constrained by $\int_{I}|\theta|^{2} d z=1$. For $\varepsilon=1 / h \ll 1$, we have

$$
\varepsilon \mu^{2} \approx 0, \quad F_{\varepsilon} \approx 1,
$$

and

$$
\int_{I}\left|\varphi_{\varepsilon}(z)-\frac{1}{\sqrt{2}}\right|^{2} d z \ll 1 .
$$


By the virtue of Theorem 5.4 and 5.6, the descriptions of the director and layer perturbations at the critical field are consistent with the result from [15] and experimental results from [28].

\section{Bifurcation}

We find an eigenfunction and an eigenvalue for the Euler-Lagrange equation of the functional $F_{\varepsilon}$ and use formal asymptotic expansions to solve the algebraic equation that occurs in the process. Furthermore, we show that the kernel of the linearized operator at the first eigenvalue is one dimensional. We also find the first eigenvalue and the corresponding eigenfunction explicitly for bifurcation study.

6.1. Linearized functional. In order to carry out a bifurcation analysis for the onset of the undulations, we find the basis of the kernel of the linearized operator at the undeformed state in this section for both boundary conditions on the layer.

\subsubsection{Dirichlet boundary condition.}

Lemma 6.1. Let $\left(\theta_{n}, \varphi_{n}\right) \in X_{d}$ be a global minimizer of $F_{\varepsilon}$ in $X_{d}$. Then $\left(\theta_{n}, \varphi_{n}\right)$ satisfies the Euler-Lagrange equations

$$
\begin{aligned}
& \frac{D_{1} \varepsilon^{2}}{\delta_{n}} \varphi_{n}^{\prime \prime \prime \prime}-\left(2 D_{1} \varepsilon+\frac{1}{\delta_{n}}\right) \varphi_{n}^{\prime \prime}+D_{1} \varepsilon \theta_{n}^{\prime \prime}+\left(D_{1} \delta_{n}+\frac{1}{\varepsilon}\right)\left(\varphi_{n}-\theta_{n}\right)=0, \\
& -\varepsilon \theta_{n}^{\prime \prime}+\delta_{n} \theta_{n}+D_{1} \varepsilon \varphi_{n}^{\prime \prime}+\left(\frac{1}{\varepsilon}+D_{1} \delta_{n}\right)\left(\theta_{n}-\varphi_{n}\right)=\sigma_{n} \theta_{n},
\end{aligned}
$$

with boundary conditions

$$
\theta_{n}( \pm 1)=0, \quad \text { and } \quad \varphi_{n}( \pm 1)=\left(\frac{\varepsilon}{\delta} \varphi_{n}^{\prime \prime}-\varphi+\theta\right)( \pm 1)=0 .
$$

There exists a discrete set $\mathcal{H} \subset \mathbb{R}$ such that for any $\varepsilon \in \mathbb{R} \backslash \mathcal{H}$ and $\sigma_{n}^{0}=\min F_{\varepsilon}\left(\theta_{n}, \varphi_{n}\right)$ in $\tilde{\mathcal{B}}$, the space of solutions to the system of equations (6.1) is one-dimensional, and is spanned by the functions

$$
\begin{aligned}
& \theta(y)=C \cos \frac{\pi}{2} y, \\
& \varphi(y)=\frac{1-\sigma_{m} \varepsilon+\sqrt{\left(1-\sigma_{m} \varepsilon\right)^{2}+4 \delta_{m} \varepsilon}}{2} \theta(y),
\end{aligned}
$$

for some constant $C$. Furthermore, $\sigma_{c}^{0}=\inf _{n} \sigma_{n}^{0}=\sigma_{m}^{0}$.

Proof. The system of Euler-Lagrange equations (6.1) can be written as

$$
\begin{gathered}
D_{1} \mathcal{D}^{2} \varphi+\frac{1}{\varepsilon} \mathcal{D} \varphi-D_{1} \delta \mathcal{D} \theta-\frac{\delta}{\varepsilon} \theta=0, \\
\mathcal{D} \theta-D_{1} \mathcal{D} \varphi+\frac{1}{\varepsilon}(\theta-\varphi)=\left(\sigma-D_{1} \delta\right) \theta,
\end{gathered}
$$

where $\mathcal{D}=-\varepsilon \frac{d^{2}}{d y^{2}}+\delta I$. Applying $\mathcal{D}$ to the second equation and combining it with the first equation, we obtain a fourth order differential equation for $\theta$,

$$
\mathcal{D}^{2} \theta+\left(\frac{1}{\varepsilon}-\sigma\right) \mathcal{D} \theta-\frac{\delta}{\varepsilon} \theta=0,
$$

which is

$$
\varepsilon^{2} \theta^{\prime \prime \prime \prime}+(-2 \varepsilon \delta+\sigma \varepsilon-1) \theta^{\prime \prime}+\delta(\delta-\sigma) \theta=0 .
$$


One can check that the following functions are solutions to this equation that are linearly independent:

$$
\begin{array}{lrl}
\theta_{1}(y) & =\sinh (\sqrt{\alpha}(y-1)), & \theta_{2}(y)=\sinh (\sqrt{\alpha}(y+1)), \\
\theta_{3}(y) & =\sin (\sqrt{\gamma}(y-1)), & \theta_{4}(y)=\sin (\sqrt{\gamma}(y+1)) .
\end{array}
$$

Here we use

$$
\alpha=\frac{\varepsilon \delta+B}{\varepsilon^{2}}, \quad \gamma=\frac{-\varepsilon \delta+A}{\varepsilon^{2}}, \quad \text { and } \quad a=1-\sigma \varepsilon
$$

when

$$
A=\frac{-a+\sqrt{a^{2}+4 \varepsilon \delta}}{2}, \quad \text { and } \quad B=\frac{a+\sqrt{a^{2}+4 \varepsilon \delta}}{2} .
$$

Therefore the general solution to $(6.5)$ is

$$
\theta(x)=\sum_{i=1}^{4} d_{i} \theta_{i}(x)
$$

From (4.15), one can see that $\sigma>\delta$ and it is equivalent to $\gamma>0$ and thus $A>\varepsilon \delta>0$ and $B>a$. Using (6.7), the second equation of (6.1) becomes

$$
\begin{aligned}
D_{1} \varepsilon \varphi^{\prime \prime}-\left(\frac{1}{\varepsilon}+D_{1} \delta\right) \varphi=( & \left.\alpha \varepsilon+\lambda-\delta-\frac{1}{\varepsilon}-D_{1} \delta\right)\left(d_{1} \theta_{1}+d_{2} \theta_{2}\right) \\
& +\left(-\gamma \varepsilon+\lambda-\delta-\frac{1}{\varepsilon}-D_{1} \delta\right)\left(d_{3} \theta_{3}+d_{4} \theta_{4}\right)
\end{aligned}
$$

Then one can find the general solution to (6.8),

$$
\varphi=b_{1} \sinh (\beta(y-1))+b_{2} \sinh (\beta(y+1))-A\left(d_{1} \theta_{1}+d_{2} \theta_{2}\right)+B\left(d_{3} \theta_{3}+d_{4} \theta_{4}\right),
$$

where

$$
\beta=\frac{1}{\varepsilon} \sqrt{\frac{1+D_{1} \delta \varepsilon}{D_{1}}}
$$

Using the boundary condition (6.2) and setting

$$
s_{1}=\sinh (2 \sqrt{\alpha}), \quad s_{2}=\sin (2 \sqrt{\gamma}), \quad \text { and } \quad s_{3}=\sinh (2 \beta),
$$

we have

$$
\left(\begin{array}{cccccc}
s_{1} & 0 & s_{2} & 0 & 0 & 0 \\
0 & s_{1} & 0 & s_{2} & 0 & 0 \\
A s_{1} & 0 & -B s_{2} & 0 & -c s_{3} & 0 \\
0 & A s_{1} & 0 & -B s_{2} & 0 & -c s_{3} \\
l_{1} s_{1} & 0 & l_{2} s_{2} & 0 & -l_{3} s_{3} & 0 \\
0 & l_{1} s_{1} & 0 & l_{2} s_{2} & 0 & -l_{3} s_{3}
\end{array}\right)
$$

where

$$
l_{1}=A\left(-\frac{\varepsilon \alpha}{\delta}+1\right)+1
$$




$$
\begin{aligned}
& l_{2}=-B\left(\frac{\varepsilon \gamma}{\delta}+1\right)+1, \\
& l_{3}=\left(\frac{\varepsilon}{\delta} \beta^{2}-1\right) .
\end{aligned}
$$

Computing this with (6.6), we find

$$
l_{1}=l_{2}=0 \quad \text { and } \quad l_{3}=\frac{1}{D_{1} \delta \varepsilon}>0 .
$$

Thus this matrix is equivalent to

$$
\left(\begin{array}{cccccc}
s_{1} & 0 & s_{2} & 0 & 0 & 0 \\
0 & s_{1} & 0 & s_{2} & 0 & 0 \\
0 & 0 & (A+B) s_{2} & 0 & 0 & 0 \\
0 & 0 & 0 & (A+B) s_{2} & 0 & 0 \\
0 & 0 & 0 & 0 & 1 & 0 \\
0 & 0 & 0 & 0 & 0 & 1
\end{array}\right) .
$$

Because $A+B=\sqrt{a^{2}+4 \delta \varepsilon}>0$ and $s_{1}>0$, we must have $s_{2}=0$, which is identical to the formula with Dirichlet boundary condition in [15]. This implies $d_{1}=d_{2}=b_{1}=$ $b_{2}=0$ and $\sqrt{\gamma}=n \pi / 2$ for some integer $n \neq 0$. Solving (6.6) for $\sigma$, we have

$$
\sigma=\frac{4 \delta+\varepsilon n^{2} \pi^{2}}{4}+\frac{n^{2} \pi^{2}}{4 \delta+\varepsilon n^{2} \pi^{2}} .
$$

Then we see that $\sigma$ is minimized when $n= \pm 1$. Hence we have

$$
\begin{aligned}
\theta(y) & =\left(-d_{3}+d_{4}\right) \cos \frac{\pi}{2} y, \\
\varphi(y) & =B \theta(y), \\
\sigma & =\sigma(\delta)=\frac{4 \delta+\varepsilon \pi^{2}}{4}+\frac{\pi^{2}}{4 \delta+\pi^{2} \varepsilon} .
\end{aligned}
$$

Differentiating $\sigma(\delta)$ with $\delta$, one can see that the minimum value of $\sigma$ is obtained when

$$
\delta=\frac{\pi}{2}-\frac{\pi^{2} \varepsilon}{4}
$$

Then the minimum value of $\sigma$ is

$$
\sigma\left(\frac{\pi}{2}-\frac{\pi^{2} \varepsilon}{4}\right)=\pi
$$

We conclude that $\theta$ and $\varphi$ in (6.12) are eigenfunctions associated with the first eigenvalue $\sigma$ in (6.12). We need to recall that the choice of $\mu$ is restricted by $\mu=\pi n / r$ for some integer $n$, where $r=\widetilde{L} / h$. Following the same analysis in [15], we conclude that the eigenvalue is simple for all $\varepsilon$ except for a discrete set and there is an integer $m$ from (6.13) such that $\sigma_{m}=\sigma\left(\delta_{m}\right)$, where $\delta_{m}=\varepsilon(\pi m / r)^{2}$ is the first eigenvalue for the system (6.1). The corresponding eigenfunctions (6.3) are then obtained from (6.12). We also note that $\varphi_{m}-\theta_{m}=\mathcal{O}(\sqrt{\varepsilon})$. 


\subsubsection{Natural boundary condition on layers.}

Lemma 6.2. A minimizer of $F_{\varepsilon}$ in $X_{n}$ is taken by real valued functions $\left(\theta_{n}, \varphi_{n}\right)$, which satisfy the system (6.1) with the boundary condition

$$
\begin{aligned}
& \theta_{n}( \pm 1)=0 \\
& \left(\frac{\varepsilon}{\delta} \varphi_{n}^{\prime \prime}-\varphi_{n}+\theta_{n}\right)( \pm 1)=\left(-D_{1} \varepsilon\left(\frac{\varepsilon}{\delta} \varphi_{n}^{\prime \prime \prime}-\varphi_{n}^{\prime}+\theta_{n}^{\prime}\right)+\frac{1}{\delta} \varphi_{n}^{\prime}\right)( \pm 1)=0 .
\end{aligned}
$$

Also, the minimizer of $F_{\varepsilon}$ is obtained when $\delta_{n}=\delta_{1}=\varepsilon(\pi)^{2}+\mathcal{O}\left(\varepsilon^{2}\right)$ and the minimum value of the functional is

$$
\sigma_{n}=\sigma_{1}=1+\left(0.5+2 / 3(\pi)^{2}\right) \varepsilon+\mathcal{O}\left(\varepsilon^{2}\right) .
$$

For $\sigma_{n}=\min F_{\varepsilon}\left(\theta_{n}, \varphi_{n}\right)$ in $\tilde{\mathcal{B}}$, the solution to the system is spanned by the one element,

$$
\begin{aligned}
& \theta_{n}(y)=-\cos \sqrt{\gamma} \cosh \sqrt{\alpha} y+\cosh \sqrt{\alpha} \cos \sqrt{\gamma} y, \\
& \varphi_{n}(y)=A \cos \sqrt{\gamma} \cosh \sqrt{\alpha} y+B \cosh \sqrt{\alpha} \cos \sqrt{\gamma} y,
\end{aligned}
$$

where $\alpha, \gamma, A$, and $B$ are given in (6.6). Furthermore, any solution of $(6.1,6.15)$ is of the form $C\left(\theta_{n}, \varphi_{n}\right)$ for some constant $C$.

Proof. As in the proof of Lemma 6.1, we have the general solutions (6.7) and (6.9). Using the boundary condition (6.15), we have

$$
\left(\begin{array}{cccccc}
s_{1} & 0 & s_{2} & 0 & 0 & 0 \\
0 & s_{1} & 0 & s_{2} & 0 & 0 \\
-A \sqrt{\alpha} c_{1} & -A \sqrt{\alpha} & \sqrt{\gamma} B c_{2} & \sqrt{\gamma} B & 0 & 0 \\
-A \sqrt{\alpha} & -A \sqrt{\alpha} c_{1} & \sqrt{\gamma} B & \sqrt{\gamma} B c_{2} & 0 & 0 \\
0 & 0 & 0 & 0 & s_{3} & 0 \\
0 & 0 & 0 & 0 & 0 & s_{3}
\end{array}\right) .
$$

Here the same notations in Lemma 6.1 are used and

$$
c_{1}=\cosh (2 \sqrt{\alpha}), \quad c_{2}=\cos (2 \sqrt{\gamma}), \quad \text { and } \quad c_{3}=\cosh (2 \beta) .
$$

We notice that the first $4 \times 4$ matrix in (6.18) is identical to the coefficient matrix found in the proof of Theorem 6 of [15] and hence the same eigenfunction and eigenvalue are obtained, which are given in (6.17).

One can notice that the results obtained in Section 5 and this section are consistent. Theorem 5.4 from the $\Gamma$-convergence method and Lemma 6.1 from the eigenvalue estimate give the same description of the director and layer undulations as well as the estimate of the critical field for the case of homogeneous Dirichlet boundary condition for the layer function. For natural boundary condition on the layer function, the same results are obtained from Theorem 5.6 and Lemma 6.2.

6.2. Bifurcation curve. Setting $\varphi=u-y$ in the energy (4.9), $\varepsilon \mathfrak{F}$ becomes, with $D=(-r, r) \times(-1,1)$,

$$
\mathcal{F}(\theta, \varphi, \sigma):=\int_{D}\left(D_{1} \varepsilon\left(\Delta \varphi-\cos \theta \theta_{x}+\sin \theta \theta_{y}\right)^{2}\right.
$$




$$
\begin{aligned}
& +\frac{D_{2} \varepsilon}{2}\left(\left(\varphi_{x}-\sin \theta\right)^{2}+\left(\varphi_{y}+1-\cos \theta\right)^{2}\right)^{2}+\varepsilon|\nabla \theta|^{2}(6.19) \\
& \left.+\frac{1}{\varepsilon}\left(\varphi_{x}-\sin \theta\right)^{2}+\frac{1}{\varepsilon}\left(\varphi_{y}+1-\cos \theta\right)^{2}-\sigma \sin ^{2} \theta\right) d y d x
\end{aligned}
$$

and $(\theta, \varphi)=(0,0)$ corresponds to the flat layer state. We define the spaces

$$
\begin{aligned}
& \mathbf{Z}=\left\{\mathbf{z}=(\theta, \varphi): \theta, \varphi \in C^{2}(\bar{U}), \theta(x, \pm 1)=\varphi(x, \pm 1)=0 \text { for all } x\right\} \\
& \mathbf{Y}=C^{0}(\bar{U}) \times C^{0}(\bar{U})
\end{aligned}
$$

with the graph topology, where $U=\mathbb{R} /(-r+2 r \mathbb{Z}) \times(-1,1)$, having the periodic boundary condition in the $x$ direction. The Euler-Lagrange equations associated with the energy (6.19) are, for $\mathbf{z}=(\theta, \varphi)$,

$$
\begin{aligned}
& F_{1}(\mathbf{z}, \sigma):=D_{1} \varepsilon \nabla\left(\Delta \varphi-\cos \theta \theta_{x}+\sin \theta \theta_{y}\right) \cdot\left(\begin{array}{c}
\cos \theta \\
-\sin \theta
\end{array}\right) \\
&+ D_{2} \varepsilon\left(\left(\varphi_{x}-\sin \theta\right)^{2}+\left(\varphi_{y}+1-\cos \theta\right)^{2}\right)\left(-\cos \theta \varphi_{x}+\sin \theta \varphi_{y}+\sin \theta\right) \\
&- \varepsilon \Delta \theta+\frac{1}{\varepsilon}\left(\begin{array}{c}
-\cos \theta \\
\sin \theta
\end{array}\right) \nabla \varphi+\frac{1}{\varepsilon} \sin \theta-\frac{\sigma}{2} \sin 2 \theta=0, \\
& F_{2}(\mathbf{z}, \sigma):=D_{1} \varepsilon \Delta\left(\Delta \varphi-\left(\begin{array}{c}
\cos \theta \\
-\sin \theta
\end{array}\right) \cdot \nabla \theta\right) \\
&-D_{2} \varepsilon \nabla \cdot\left(\left(\left(\varphi_{x}-\sin \theta\right)^{2}+\left(\varphi_{y}+1-\cos \theta\right)^{2}\right)\left(\begin{array}{c}
\varphi_{x}-\sin \theta \\
\varphi_{y}+1-\cos \theta
\end{array}\right)\right) \\
&-\frac{1}{\varepsilon}\left(\Delta \varphi-\left(\begin{array}{c}
\cos \theta \\
-\sin \theta
\end{array}\right) \nabla \theta\right)=0 .
\end{aligned}
$$

We let $\mathbf{F}=\left(F_{1}, F_{2}\right): \mathbf{Z} \times \mathbb{R} \rightarrow \mathbf{Y}$. Recall that $\sigma_{c}^{0}$ is the minimum of the linearized functional at the undeformed state under the Dirichlet boundary conditions for both $\theta$ and $\varphi$. From Lemma 6.1, we have for $\sigma=\sigma_{c}^{0}$, and except for a discrete set for $\varepsilon$, that the linearized problem

$$
\begin{aligned}
& -D_{1} \varepsilon\left(\Delta \varphi_{x}-\theta_{x x}\right)+\frac{1}{\varepsilon}\left(\varphi_{x}-\theta\right)+\varepsilon \Delta \theta+\sigma \theta=0, \\
& D_{1} \varepsilon \Delta\left(\Delta \varphi-\theta_{x}\right)-\frac{1}{\varepsilon} \Delta \varphi+\frac{1}{\varepsilon} \theta_{x}=0
\end{aligned}
$$

has a solution set spanned by

$$
\mathbf{z}_{1}=\left(\theta_{1}, \varphi_{1}\right):=\left(\theta_{m}(y) \cos \mu_{m} x, \varphi_{m}(y) \sin \mu_{m} x\right)
$$

for some $m$. Reformulating these equations in Fourier space and using (4.13) and Lemma 6.1, one can find that

$$
\begin{aligned}
\theta_{1} & =\cos \frac{\pi}{2} y \cos \mu_{m} x \\
\varphi_{1} & =\frac{1-\sigma_{m} \varepsilon+\sqrt{\left(1-\sigma_{m} \varepsilon\right)^{2}+4 \delta_{m} \varepsilon}}{2 \mu_{m}} \cos \frac{\pi}{2} y \sin \mu_{m} x .
\end{aligned}
$$

As with the natural boundary condition (6.15) on $\varphi$, for $\sigma=\sigma_{c}$, the linearized problem (6.22) has a solution set spanned by

$$
\mathbf{z}_{1}=\left(\theta_{1}, \varphi_{1}\right):=\left(u(y) \cos \mu_{1} x, \frac{v(y)}{\mu_{1}} \sin \mu_{1} x\right),
$$


where

$$
\begin{aligned}
& u(y)=-\cos \sqrt{\gamma} \cosh \sqrt{\alpha} y+\cosh \sqrt{\alpha} \cos \sqrt{\gamma} y, \\
& v(y)=A \cos \sqrt{\gamma} \cosh \sqrt{\alpha} y+B \cosh \sqrt{\alpha} \cos \sqrt{\gamma} y .
\end{aligned}
$$

Then $\sigma_{c}^{0}$ and $\sigma_{c}$ are simple eigenvalues and the structure of the local solution curves can be understood using the Crandall-Rabinowitz theory for bifurcations at simple eigenvalues $([4])$.

Theorem 6.3. At $\sigma=\sigma_{c}^{0}$ and $\sigma_{c}$ for each boundary condition, there is an $r>0$ and bifurcation curve of solutions to the system (6.20, 6.21) for $s \in(-r, r)$, satisfying

$$
\begin{aligned}
& \text { 1. } \mathbf{F}(\mathbf{z}(s), \sigma(s))=0, \\
& \text { 2. }(\mathbf{z}(0), \sigma(0))=\left(0, \sigma_{c}^{0}\right), \\
& \text { 3. } \mathbf{z}^{\prime}(0)=\left(\theta_{1}, \varphi_{1}\right), \\
& \text { 4. } \theta(s)=s \theta_{1}+\mathcal{O}\left(s^{2}\right), \varphi(s)=s \varphi_{1}+\mathcal{O}\left(s^{2}\right) \text { and } \sigma(s)=\sigma_{c}^{0}+\mathcal{O}\left(s^{2}\right) \text {. }
\end{aligned}
$$

Furthermore, the system (6.20, 6.21) has only two solutions, $(\mathbf{z}(s), \sigma(s))$ and $\mathbf{z}=0$, and the nontrivial solution is stable in a sufficiently small neighborhood of $\left(0, \sigma_{c}^{0}\right)$ and $\left(0, \sigma_{c}\right)$.

Proof. We follow the proof given in Theorem 5 of [15] and thus here we summarize the proof briefly. Following the Crandall-Rabinowitz theory, the existence of such a bifurcation curve can be proved in exactly the same way as in [15]. Then $\left(0, \sigma_{c}^{0}\right)$ is a bifurcation point and there is a one parameter family of nontrivial solutions $(\theta(s), \varphi(s), \sigma(s))=\left(s \theta_{1}+\theta_{2}(s), s \varphi_{1}+\varphi_{2}(s), \sigma_{c}^{0}+s \sigma_{1}\right)$.

We proceed to study the stability of the nontrivial solution curve. From Theorem 1.16 in [5] it follows that there are eigenvalues $\mu(s)$ and $\gamma(\sigma)$ with eigenvectors $\omega(s)$ and $u(\sigma)$, respectively, such that

$$
\begin{aligned}
& \mathbf{F}_{\mathbf{z}}(\mathbf{z}(s), \sigma(s)) \omega(s)=\mu(s) \omega(s), \\
& \mathbf{F}_{\mathbf{z}}(0,0, \sigma) u(\sigma)=\gamma(\sigma) u(\sigma),
\end{aligned}
$$

with $\mu(0)=\gamma\left(\sigma_{c}^{0}\right)=0$ and $\omega(0)=\left(\theta_{1}, \varphi_{1}\right)=u\left(\sigma_{c}^{0}\right)$. In order to show $\mu(s)<0$, which means stability of the nontrivial curve, we use the relation ([5])

$$
\gamma^{\prime}\left(\sigma_{c}^{0}\right) \neq 0 \quad \text { and } \quad \lim _{s \rightarrow 0} \frac{-s \sigma^{\prime}(s) \gamma^{\prime}\left(\sigma_{c}^{0}\right)}{\mu(s)}=1,
$$

if $\mu(s) \neq 0$ for $s \neq 0$. Because the codimension of the range of $\left(\mathbf{F}_{\mathbf{z}}\left(0,0, \sigma_{c}^{0}\right)\right)$ is 1 , there exists $l \in Y^{*}$ such that $N(l)=\mathcal{R}\left(\mathbf{F}_{\mathbf{z}}\left(0,0, \sigma_{c}^{0}\right)\right)$. Differentiating (6.28), we obtain that

$$
\left\langle l, \mathbf{F}_{\mathbf{z} \sigma}\left(0,0, \sigma_{c}^{0}\right) \mathbf{z}_{1}\right\rangle=\gamma^{\prime}\left(\sigma_{c}^{0}\right)\left\langle l, \mathbf{z}_{1}\right\rangle .
$$

A direct computation gives

$$
\mathbf{F}_{\mathbf{z} \sigma}\left(0,0, \sigma_{c}^{0}\right)\left(\theta_{1}, \varphi_{1}\right)=\left(\theta_{1}, 0\right),
$$

and hence

$$
\gamma^{\prime}\left(\sigma_{c}^{0}\right)>0
$$

It is not surprising to see that $\sigma^{\prime}(0)=0$ because the linearized operator is invariant with respect to $(\theta, \varphi) \rightarrow(-\theta,-\varphi)$ transformation. The proof is below. Then $\sigma^{\prime}(s)=$ 
$\sigma^{\prime \prime}(0) s+\mathcal{O}\left(s^{2}\right)$ and hence the formula (6.29) gives $\mu(s)<0$ if $\sigma^{\prime \prime}(0)>0$. Thus, Theorem 6.3 follows from the following lemma and this is a supercritical pitchfork bifurcation.

Lemma 6.4. $\sigma^{\prime}(0)=0$ and $\sigma^{\prime \prime}(0)>0$.

Proof. The derivative of $\sigma$ can be evaluated using the formula [24]

$$
\sigma^{\prime}(0)=-\frac{1}{2} \frac{\left\langle\mathbf{F}_{\mathbf{z z}}\left(0,0, \sigma_{c}^{0}\right)\left(\mathbf{z}_{1}, \mathbf{z}_{1}\right),\left(\mathbf{z}_{1}\right)\right\rangle}{\left\langle\mathbf{F}_{\mathbf{z} \sigma}\left(0,0, \sigma_{c}^{0}\right) \mathbf{z}_{1}, \mathbf{z}_{1}\right\rangle}
$$

Differentiating (6.20) twice, we find

$$
\begin{aligned}
& D_{\mathbf{z z}} F_{1}\left(\mathbf{z}_{0}, \sigma\right)[\mathbf{z}]=-D_{1} \varepsilon\left(2 \theta\left(-\Delta \varphi_{y}+2 \theta_{x y}\right)+2 \theta_{x} \theta_{y}\right)-\frac{2}{\varepsilon} \theta \varphi_{y}, \\
& D_{\mathbf{z z}} F_{2}\left(\mathbf{z}_{0}, \sigma\right)[\mathbf{z}]=-2 D_{1} \varepsilon\left(3 \theta_{y} \theta_{y y}+\theta_{x x} \theta_{y}+2 \theta_{x} \theta_{x y}+\theta \Delta \theta_{y}\right)+\frac{2}{\varepsilon} \theta \theta_{y} .
\end{aligned}
$$

Combining this with (6.33) and (6.31), we have

$$
\begin{aligned}
& \sigma^{\prime}(0)=\frac{1}{2} \int_{D}\left(D _ { 1 } \varepsilon \left[2 \theta_{1}^{2}\left(-\Delta\left(\varphi_{1}\right)_{y}+2\left(\theta_{1}\right)_{x y}\right)+2\left(\theta_{1}\right)_{x}\left(\theta_{1}\right)_{y} \theta_{1}\right.\right. \\
&\left.-2\left\{3\left(\theta_{1}\right)_{y}\left(\theta_{1}\right)_{y y}+\left(\theta_{1}\right)_{x x}\left(\theta_{1}\right)_{y}+2\left(\theta_{1}\right)_{x}\left(\theta_{1}\right)_{x y}+\theta_{1} \Delta\left(\theta_{1}\right)_{y}\right\} \varphi_{1}\right] \\
&\left.+\frac{2}{\varepsilon}\left(\theta_{1}^{2}\left(\varphi_{1}\right)_{y}-\theta_{1} \varphi_{1}\left(\theta_{1}\right)_{y}\right)\right) d x d y \\
&=0,
\end{aligned}
$$

because the integrand is an odd function in $x$ from (6.24) and (6.26). Then the formula for $\sigma^{\prime \prime}(0)$ is given by [24]

$$
\sigma^{\prime \prime}(0)=-\frac{1}{3} \frac{\left\langle\mathbf{F}_{\mathbf{z z z}}\left(0,0, \sigma_{c}^{0}\right)\left(\mathbf{z}_{1}, \mathbf{z}_{1}, \mathbf{z}_{1}\right), \mathbf{z}_{1}\right\rangle}{\left\langle\mathbf{F}_{\mathbf{z} \sigma}\left(0,0, \sigma_{c}^{0}\right) \mathbf{z}_{1}, \mathbf{z}_{1}\right\rangle} .
$$

From a direct computation, we have

$$
\begin{aligned}
D_{\mathbf{z z z}} F_{1}\left(\mathbf{z}_{0}, \sigma\right)[\mathbf{z}]=- & 3 D_{1} \varepsilon \theta_{1}\left[\theta_{1}\left(-\Delta\left(\varphi_{1}\right)_{x}+2\left(\theta_{1}\right)_{x x}-2\left(\theta_{1}\right)_{y y}\right)+2\left(\theta_{1}\right)_{x}^{2}-\left(\theta_{1}\right)_{y}^{2}\right] \\
& +6 D_{2} \varepsilon\left(\left(\varphi_{1}\right)_{x}-\theta_{1}\right)\left(\left(\left(\varphi_{1}\right)_{x}-\theta_{1}\right)^{2}+\left(\varphi_{1}\right)_{y}^{2}\right) \\
& +\frac{1}{\varepsilon} \theta_{1}^{2}\left(-3\left(\varphi_{1}\right)_{x}+\theta_{1}\right)-4 \sigma \theta_{1}^{3}, \\
D_{\mathbf{z z z}} F_{2}\left(\mathbf{z}_{0}, \sigma\right)[\mathbf{z}]=- & 3 D_{1} \varepsilon\left[2\left(\left(\theta_{1}\right)_{x}^{2}+\left(\theta_{1}\right)_{y}^{2}\right)\left(\theta_{1}\right)_{x}+\theta_{1}^{2} \Delta\left(\theta_{1}\right)_{x}\right. \\
& \left.+2 \theta_{1}\left(3\left(\theta_{1}\right)_{x}\left(\theta_{1}\right)_{x x}+\left(\theta_{1}\right)_{x}\left(\theta_{1}\right)_{y y}+2\left(\theta_{1}\right)_{y}\left(\theta_{1}\right)_{x y}\right)\right] \\
+ & 6 D_{2} \varepsilon\left[\left(\left(\varphi_{1}\right)_{x}+\left(\varphi_{1}\right)_{y}-\theta_{1}\right)^{2}\left(\Delta \varphi_{1}-\left(\theta_{1}\right)_{x}\right)\right. \\
& \left.+2\left(\left(\varphi_{1}\right)_{x}-\theta_{1}\right)^{2}\left(\left(\varphi_{1}\right)_{x x}-\left(\theta_{1}\right)_{x}\right)+2\left(\varphi_{1}\right)_{x}^{2}\left(\varphi_{1}\right)_{y y}\right] \\
+ & +\frac{3}{\varepsilon} \theta_{1}^{2}\left(\theta_{1}\right)_{x} .
\end{aligned}
$$

Using this in (6.36), we obtain

$$
\sigma^{\prime \prime}(0)=-\frac{1}{3} \int_{D}\left(-3 D_{1} \varepsilon g_{1}+6 D_{2} \varepsilon g_{2}+\frac{1}{\varepsilon} g_{3}-4 \sigma \theta_{1}^{4}\right) d x d y,
$$


where

$$
\begin{aligned}
g_{1}=\theta_{1}^{3} & \left(-\Delta \varphi_{1}+2\left(\theta_{1}\right)_{x x}-\left(\theta_{1}\right)_{y y}\right)+2 \theta_{1}^{2}\left(\theta_{1}\right)_{x}^{2}-\theta_{1}^{2}\left(\theta_{1}\right)_{y}^{2} \\
& +2 \varphi_{1}\left(\theta_{1}\right)_{x}\left(\left(\theta_{1}\right)_{x}^{2}+\left(\theta_{1}\right)_{y}^{2}\right)+\theta_{1}^{2} \varphi_{1} \Delta\left(\theta_{1}\right)_{x} \\
& +2 \theta_{1} \varphi_{1}\left(3\left(\theta_{1}\right)_{x}\left(\theta_{1}\right)_{x x}+\left(\theta_{1}\right)_{x}\left(\theta_{1}\right)_{y y}+2\left(\theta_{1}\right)_{y}\left(\theta_{1}\right)_{x y}\right) \\
g_{2}=\theta_{1} & \left(\left(\varphi_{1}\right)_{x}-\theta_{1}\right)\left(\left(\left(\varphi_{1}\right)_{x}-\theta_{1}\right)^{2}+\left(\varphi_{1}\right)^{2}\right) \\
& +\left(\left(\varphi_{1}\right)_{x}+\left(\varphi_{1}\right)_{y}-\theta_{1}\right)^{2}\left(\Delta \varphi_{1}-\left(\theta_{1}\right)_{x}\right) \varphi_{1} \\
& +2 \varphi_{1}\left(\left(\varphi_{1}\right)_{x}-\theta_{1}\right)^{2}\left(\left(\varphi_{1}\right)_{x x}-\left(\theta_{1}\right)_{x}\right)+2 \varphi_{1}\left(\varphi_{1}\right)_{x}^{2}\left(\varphi_{1}\right)_{y y}, \\
g_{3}=( & \left.-3 \theta_{1}^{2}\left(\varphi_{1}\right)_{x}+\theta_{1}^{3}\right) \theta_{1}+3 \theta_{1}^{2}\left(\theta_{1}\right)_{x} \varphi_{1} .
\end{aligned}
$$

Integrating by parts, we find

$$
\begin{aligned}
\int_{D} g_{3} d x d y & =\int_{D}\left(-3 \theta_{1}^{3}\left(\varphi_{1}\right)_{x}+\theta_{1}^{4}+3 \theta_{1}^{2}\left(\theta_{1}\right)_{x} \varphi_{1}\right) d x d y \\
& =\int_{D}\left(\theta_{1}^{4}-4 \theta_{1}^{3}\left(\varphi_{1}\right)_{x}\right) d x d y
\end{aligned}
$$

With the Dirichlet boundary condition, and using (6.24), (6.39) becomes

$$
\int_{D}(1-4 A) \theta_{1}^{4} d x d y
$$

where $A=\frac{1}{2}\left(1-\sigma_{m} \varepsilon+\sqrt{\left(1-\sigma_{m} \varepsilon\right)^{2}+4 \delta_{m} \varepsilon}\right)=1+\mathcal{O}(\sqrt{\varepsilon})$. Thus $g_{3}<0$. With the natural boundary condition, and using (6.26), (6.39) becomes

$$
\begin{aligned}
g_{3} & =u^{3} \cos ^{4} \mu_{1} x(u-4 v) \\
& =u^{3} \cos ^{4} \mu_{1} x((1-4 B) u-4(A+B) \cos \sqrt{\gamma} \cosh (\sqrt{\alpha} y)) .
\end{aligned}
$$

From the estimate that $A=\mathcal{O}(\varepsilon), B \sim c, \gamma \sim 0$, we have $g_{3}<0$. Note that $g_{1}$ and $g_{2}$ are $\mathcal{O}\left(\varepsilon^{-1}\right)$ because $\mu_{m}^{2}=\mathcal{O}\left(\frac{1}{\varepsilon}\right)$. From (6.38) and the estimate $\sigma_{c}^{0}=\mathcal{O}(1)$ and $\sigma_{c}^{0}=\mathcal{O}(1)$, it is easy to see that $\sigma^{\prime \prime}(0)>0$ as claimed.

\section{Asymptotic analysis of a domain wall: smectic a energy}

In this section, we consider one dimensional domain wall profile by using asymptotic analysis for the applied magnetic field $\kappa \geq 1$. This process is motivated by the work in [12]. From $|\mathbf{n}|=1$, we can introduce the scalar variable $\theta$, with $0 \leq \theta<2 \pi$, such that

$$
\mathbf{n}=(\sin \theta, \cos \theta)
$$

and $\theta=\theta(x)$ and $\phi=\phi(x), \theta \rightarrow \pm \pi / 2$ as $x \rightarrow \pm \infty$. Then the free energy (3.4) becomes

$$
\begin{aligned}
\mathfrak{F}(\theta, \phi)=\int_{\mathbb{R}}\left(D _ { 1 } \left(\phi^{\prime \prime}\right.\right. & \left.-\cos \theta \theta^{\prime}\right)^{2}+\frac{D_{2}}{2}\left(\left(\phi^{\prime}-\sin \theta\right)^{2}+\cos ^{2} \theta\right)^{2} \\
& \left.+\left(\left(\phi^{\prime}-\sin \theta\right)^{2}+\cos ^{2} \theta\right)+\theta^{\prime 2}-\kappa^{2} \sin ^{2} \theta\right) d x
\end{aligned}
$$

The energy is minimized if

$$
\phi^{\prime}=\sin \theta
$$


Then the Euler-Lagrange equation is

$$
\theta^{\prime \prime}+\left(D_{2} \cos ^{2} \theta+1+\kappa^{2}\right) \sin \theta \cos \theta=0 .
$$

As in [12], we write a unit vector $\mathbf{n}$ as $\mathbf{n}=(\tanh \zeta, \operatorname{sech} \zeta)$ rather than (7.1) and then find the equation for $\zeta$. From $\sin \theta=\tanh \zeta$, we obtain

$$
\theta^{\prime \prime}=\operatorname{sech} \zeta\left(\zeta^{\prime \prime}-\zeta^{\prime 2} \tanh \zeta\right)
$$

and the system (7.4) becomes

$$
\begin{aligned}
& \zeta^{\prime \prime}+\tanh \zeta\left(D_{2} \operatorname{sech}^{2} \zeta-\zeta^{\prime 2}\right)+\left(1+\kappa^{2}\right) \tanh \zeta=0, \\
& \phi^{\prime}=\tanh \zeta .
\end{aligned}
$$

By changing a variable, $\tilde{x}=\kappa x, \zeta(x)=\varphi(\tilde{x})$ and $\phi(x)=\eta(\tilde{x}),(7.5)$ becomes

$$
\begin{aligned}
& \varphi^{\prime \prime}-\tanh \varphi \varphi^{\prime 2}+\tanh \varphi+\varepsilon^{2}\left(D_{2} \operatorname{sech} \varphi+1\right) \tanh \varphi=0, \\
& \eta^{\prime}=\varepsilon \tanh \varphi
\end{aligned}
$$

where $\varepsilon=1 / \kappa$. Assuming $\varphi=\varphi_{0}+\varepsilon \varphi_{1}+\varepsilon^{2} \varphi_{2}+\cdots$, and $\eta=\eta_{0}+\varepsilon \eta_{1}+\varepsilon^{2} \eta_{2}+\cdots$, we obtain the $\mathcal{O}(1)$ system:

$$
\begin{aligned}
& \varphi_{0}^{\prime \prime}+\left(1-\left(\varphi_{0}^{\prime}\right)^{2}\right) \tanh \varphi_{0}=0 \\
& \eta_{0}^{\prime}=0 .
\end{aligned}
$$

The first equation corresponds to the equation $\theta_{0}^{\prime \prime}+\sin \theta_{0} \cos \theta_{0}=0$. It is known that $\sin \theta_{0}=\tanh x$ is a solution, which describes the Landau-Lifshitz profile $([12,25])$. Thus, the solution to (7.7) is $\varphi_{0}=\tilde{x}$ and $\eta_{0}=c_{0}$ for some constant $c_{0}$. Now the system of the order $\mathcal{O}(\varepsilon)$ is

$$
\begin{aligned}
& \varphi_{1}^{\prime \prime}-2 \varphi_{0}^{\prime} \varphi_{1}^{\prime} \tanh \varphi_{0}-\left(\varphi_{0}^{\prime}\right)^{2} \varphi_{1} \operatorname{sech}^{2} \varphi_{0}+\varphi_{1} \operatorname{sech}^{2} \varphi_{0}=0 \\
& \eta_{1}^{\prime}=\tanh \varphi_{0} .
\end{aligned}
$$

Because $\varphi_{0}=\tilde{x},(7.8)$ becomes

$$
\varphi_{1}^{\prime \prime}-2 \varphi_{1}^{\prime} \tanh \tilde{x}=0 \quad \text { and } \quad \eta_{1}^{\prime}=\tanh \tilde{x} .
$$

As in [12],

$$
\left(\frac{\varphi_{1}^{\prime}}{\cosh ^{2} \tilde{x}}\right)^{\prime}=\frac{\varphi_{1}^{\prime \prime}-2 \varphi_{1}^{\prime} \tanh \tilde{x}}{\cosh ^{2} \tilde{x}}=0
$$

gives

$$
\varphi_{1}(\tilde{x})=b_{1} \frac{\sinh 2 \tilde{x}+2 \tilde{x}}{4}+b_{2} .
$$

We have $b_{2}=0$ from the assumption that $\varphi$ is odd and $b_{1}=0$ so that $\varphi_{0}$ dominates $\varphi_{1}$. Thus, we have $\varphi_{1}=0$. The solution $\eta_{1}$ is given by

$$
\eta_{1}=\int \tanh \tilde{x} d \tilde{x}=-\ln (\cosh \tilde{x})+c_{1}
$$


The $\mathcal{O}\left(\varepsilon^{2}\right)$ system is

$$
\begin{aligned}
\varphi_{2}^{\prime \prime}-\tanh \varphi_{0}\left(2 \varphi_{0}^{\prime} \varphi_{2}^{\prime}+\varphi_{1}^{\prime 2}\right)-2 \varphi_{0}^{\prime} \varphi_{1}^{\prime} \varphi_{1} \operatorname{sech}^{2} \varphi_{0} & \\
-\left(\varphi_{2} \operatorname{sech}^{2} \varphi_{0}-\varphi_{1}^{2} \operatorname{sech}^{2} \varphi_{0} \tanh \varphi_{0}\right) \varphi_{0}^{\prime 2}+\varphi_{2} \operatorname{sech}^{2} \varphi_{0} & \\
-\varphi_{1}^{2} \operatorname{sech}^{2} \varphi_{0} \tanh \varphi_{0}+\left(D_{2} \operatorname{sech}^{2} \varphi_{0}+1\right) \tanh \varphi_{0} & =0 \\
\eta_{2}^{\prime} & =\varphi_{1} \operatorname{sech}^{2} \varphi_{0} .
\end{aligned}
$$

Using $\varphi_{0}=\tilde{x}$ and $\varphi_{1}=0,(7.10)$ becomes

$$
\varphi_{2}^{\prime \prime}-2 \varphi_{2}^{\prime} \tanh \tilde{x}+\left(D_{2} \operatorname{sech}^{2} \tilde{x}+1\right) \tanh \tilde{x}=0 \quad \text { and } \quad \eta_{2}^{\prime}=0 .
$$

By following the calculation in [12], the solution can be found to be

$$
\varphi_{2}=\frac{D_{2}}{4} \tanh \tilde{x}+\frac{1}{2} \tilde{x} \quad \text { and } \quad \eta_{2}=c_{2} .
$$

Thus, for $\varepsilon \ll 1$,

$$
\begin{aligned}
& \varphi(\tilde{x})=\tilde{x}+\frac{\varepsilon^{2}}{4}\left(D_{2} \tanh \tilde{x}+2 \tilde{x}\right)+\mathcal{O}\left(\varepsilon^{3}\right), \\
& \eta(\tilde{x})=c_{0}+\varepsilon\left(-\ln (\cosh (\tilde{x}))+c_{1}\right)+\varepsilon^{2} c_{2}+\mathcal{O}\left(\varepsilon^{3}\right),
\end{aligned}
$$

and then for $\kappa \gg 1$,

$$
\begin{aligned}
& \zeta(x)=\kappa x+\frac{1}{4 \kappa^{2}}\left(D_{2} \tanh (\kappa x)+2 \kappa x\right)+\mathcal{O}\left(\frac{1}{\kappa^{3}}\right) . \\
& \phi(x)=-\frac{1}{\kappa} \ln (\cosh (\kappa x))+c_{0}+\frac{c_{1}}{\kappa}+\frac{c_{2}}{\kappa^{2}}+\mathcal{O}\left(\frac{1}{\kappa^{3}}\right) .
\end{aligned}
$$

Therefore, we have

$$
\mathbf{n}=\left(\tanh \left(\kappa x+\frac{D_{2} \tanh (\kappa x)+2 \kappa x}{4 \kappa^{2}}\right), \operatorname{sech}\left(\kappa x+\frac{D_{2} \tanh (\kappa x)+2 \kappa x}{4 \kappa^{2}}\right)\right)+\mathcal{O}\left(\kappa^{-3}\right) .
$$
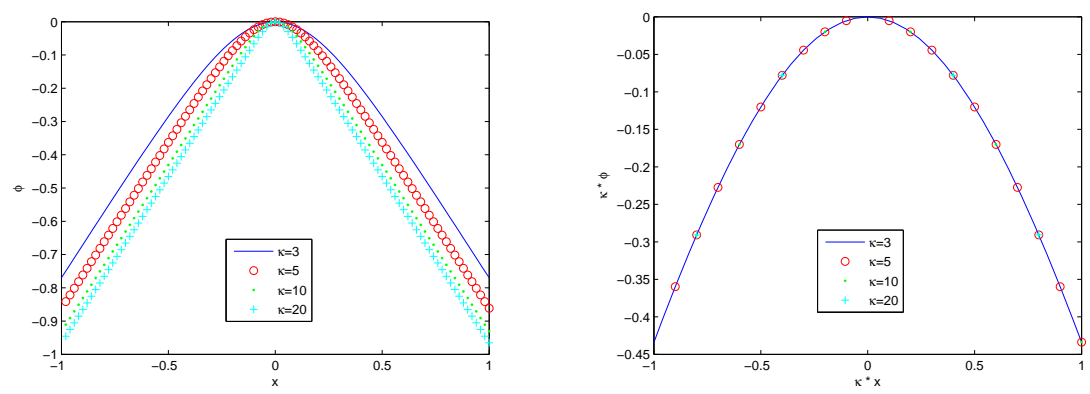

FIG. 7.1. The first column depicts the profile of $\phi$ in the variation of $\kappa$. The profile and abscissa are scaled by $\kappa$ in the second column.

The profiles (7.11) of $\phi$ in the variation of $\kappa$ with $c_{0}=1$ and $c_{1}=c_{2}=0$ are given in figure 7.1. We also illustrate layers scaled by $\kappa$. This suggests the profile of the layer variable when $\kappa \gg 1$. The asymptotic solutions of $\mathbf{n}$ are depicted in the first column of figure 7.2 and 

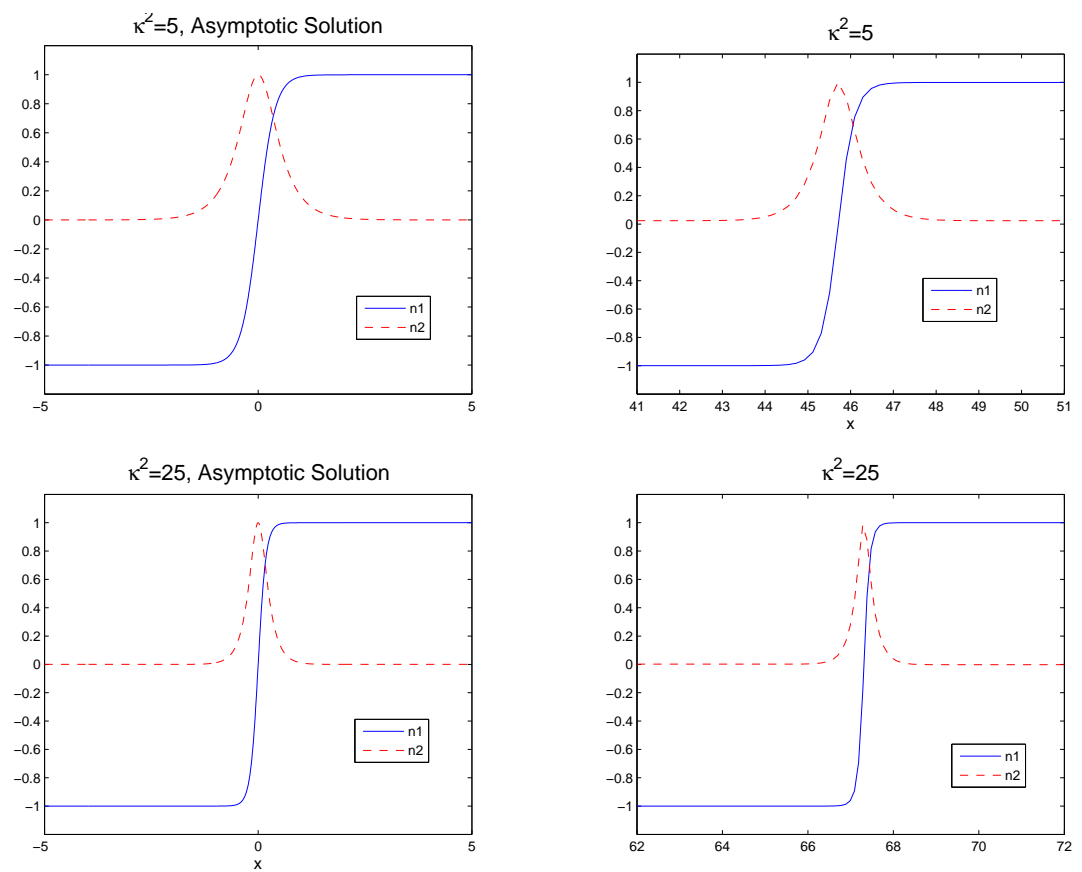

FIG. 7.2. The asymptotic approximation obtained in (7.12) and numerical solution profile in the middle of the cell.

the director profiles in the middle of the cell $(y=0)$ numerically computed in the Section 3 are presented in the second column with $\kappa^{2}=5$ and 25. Even though the description in Section 3 is governed by the Dirichlet boundary condition on the top and bottom of the cell, one can see that the domain wall profile is well approximated by the asymptotic solution where we have an infinite one dimensional problem.

\section{REFERENCES}

[1] D.L. Brown, R. Cortez, and M.L. Minion, Accurate projection methods for the incompressible Navier-Stokes equations, J. Comput. Phys., 168(2), 464-499, 2001.

[2] J. Chen and T.C. Lubensky, Landau-Ginzburg mean-field theory for the nematic to smectic-C and nematic to smectic-A phase transitions, Phys. Rev. A, 14, 1202-1207, 1976.

[3] A.J. Chorin, Numerical solution of the Navier-Stokes equations, Math. Comput., 22, 745-762, 1968.

[4] M.G. Crandall and P.H. Rabinowitz, Bifurcation from simple eigenvalues, J. Funct. Anal., 8(2), 321-340, 1971.

[5] M.G. Crandall and P.H. Rabinowitz, Bifurcation, perturbation of simple eigenvalues, and linearized stability, Arch. Rat. Mech. Anal., 52, 161-180, 1973. 10.1007/BF00282325.

[6] G. Dal Maso, Introduction to $\Gamma$-Convergence, Progress in Nonlinear Differential Equations and Their Applications, Birkhäuser, 1993.

[7] P.G. de Gennes, An analogy between superconductors and smectics A, Solid State Commun., 10(9), 753-756, 1972.

[8] P.G. de Gennes, The Physics of Liquid Crystals, International Series of Monographs on Physics, Clarendon Press, 1974.

[9] W. E and J.G. Liu, Gauge method for viscous incompressible flows, Commun. Math. Sci., 1(2), 317-332, 2003. 
[10] W. E and X.P. Wang, Numerical methods for the Landau-Lifshitz equation, SIAM J. Numer. Anal., 38(5), 1647-1665, 2000.

[11] M. Frigo and S.G. Johnson, The design and implementation of FFTW3, Proc. IEEE, 93(2), 216-231, 2005.

[12] C.J. García-Cervera, Neel walls in low anisotropy symmetric double layers, SIAM J. Appl. Math., 65(5), 1726-1747, 2005.

[13] C.J. García-Cervera, Numerical micromagnetics: A review, Bol. Soc. Esp. Mat. Apl. SẻMA, (39), 103-135, 2007.

[14] C.J. García-Cervera and S. Joo, Layer undulations in smectic A liquid crystals, J. Comput. Theor. Nanoscience, 7(4), 795-801, 2010.

[15] C.J. García-Cervera and S. Joo, Analytic description of layer undulations in smectic A liquid crystals, Arch. Rat. Mech. Anal., 203(1), 1-43, 2012.

[16] J.L. Guermond, P. Minev, and J. Shen, An overview of projection methods for incompressible flows, Comput. Meth. Appl. Mech. Engrg., 195(44-47), 6011-6045, 2006.

[17] J.L. Guermond and J. Shen, A new class of truly consistent splitting schemes for incompressible flows, J. Comput. Phys., 192(1), 262-276, 2003.

[18] W. Helfrich, Electrohydrodynamic and dielectric instabilities of cholesteric liquid crystals, J. Chemical Physics, 55(2), 839-842, 1971.

[19] A. Hubert and R. Schäfer, Magnetic Domains: The Analysis of Magnetic Microstructures, Springer-Verlag, Berlin-Heidelberg-New York, 1998.

[20] J.P. Hurault, Static distortions of a cholesteric planar structure induced by magnetic or ac electric fields, J. Chem. Phys., 59(4), 2068-2075, 1973.

[21] T. Ishikawa and O.D. Lavrentovich, Undulations in a confined lamellar system with surface anchoring, Phys. Rev. E, 63, 030501, 2001.

[22] S. Joo and D. Phillips, The phase transitions from chiral nematic toward smectic liquid crystals, Commun. Math. Phys., 269(2), 369-399, 2007.

[23] W.F. Brown Jr, Micromagnetics, Interscience Tracts on Physics and Astronomy. Interscience Publishers (John Wiley and Sons), New York - London, 1963.

[24] H. Kielhöfer, Bifurcation Theory, Appl. Math. Sci., Springer-Verlag, New York, 156, 2004.

[25] L. Landau and E. Lifshita, On the theory of the dispersion of magnetic permeability in ferromagnetic bodies, Phys. Z. Sowjetunion, 8, 153-169, 1935.

[26] J.G. Liu, J. Liu, and R.L. Pego, Stability and convergence of efficient Navier-Stokes solvers via a commutator estimate, Commun. Pure Appl. Math., 60(10), 1443-1487, 2007.

[27] I. Luk'yanchuk, Phase transition between the cholesteric and twist grain boundary $C$ phases, Phys. Rev. E, 57, 574-581, 1998.

[28] B.I. Senyuk, I.I. Smalyukh, and O.D. Lavrentovich, Undulations of lamellar liquid crystals in cells with finite surface anchoring near and well above the threshold, Phys. Rev. E, 74, $011712,2006$.

[29] I.W. Stewart, The Static and Dynamic Continuum Theory of Liquid Crystals, Taylor and Francis, 2004.

[30] M. Struwe, Heat-flow methods for harmonic maps of surfaces and applications to free boundary problems, in Fernando Cardoso, Djairo G. Figueiredo, Rafael Irio, and Orlando Lopes, editors, Part. Diff. Eqs., Lecture Notes in Mathematics, Springer Berlin Heidelberg, 1324, 293-319, 1988.

[31] R. Temam, Navier-Stokes Equations, Stud. Math. Appl., North-Holland Publishing Co., Amsterdam, Third Edition, 1984.

[32] N. Vaupotič, V. Grubelnik, and M. Čopič, Influence of an external electric field on structure in surface-stabilized smectic-C chevron cells, Phys. Rev. E, 62, 2317-2323, 2000.

[33] C. Wang and J.-G. Liu, Convergence of gauge method for incompressible flow, Math. Comput., 69(232), 1385-1407, 2000. 